\title{
ACTUALIZACIÓN AL LISTADO DE HIMENÓPTEROS (INSECTA: HYMENOPTERA) DE LA REGIÓN PIURA, PERÚ
}

\author{
Gino Juárez-Noé ${ }^{1, *}$ \\ ${ }^{1}$ Distrito de Piura, Región de Piura-Perú. \\ Email: norbiol@hotmail.com - ORCID iD: https://orcid.org/0000-0002-8847-6059 \\ *Corresponding author: norbiol@hotmail.com
}

\begin{abstract}
RESUMEN
Con base en colectas, registros bibliográficos y revisión de material depositado en colecciones entomológicas se realiza una actualización al listado de himenópteros de la región Piura al noroeste de Perú. Se adicionan 22 especies, 18 géneros, cuatro familias y se completa la identificación hasta el nivel de especie de nueve taxones citados en el listado previo, quedando la lista actualizada en 167 especies, 131 géneros, 30 familias y nueve superfamilias. Para 18 especies ya conocidas se reportan nuevos datos provinciales ampliando su área de distribución conocida dentro de la región. Las especies añadidas en esta actualización se presentan a modo de listado taxonómico comentado y se incluye lista actualizada de todos los himenópteros conocidos hasta el momento para la región con distribución por provincia y paisaje ecológico.
\end{abstract}

Palabras clave: Hymenoptera, actualización, distribución, paisaje ecológico, región Piura, Perú.

\section{ABSTRACT}

Update to the checklist of hymenopterans (Insecta: Hymenoptera) of Piura region, Peru

An update to the checklist of hymenopterans of the Piura region in northwestern Peru is made based on specimen collecting, bibliographic records and a review of the material deposited in entomological collections. A total of 22 species, 18 genera and four families are added and we complete the identification to species level for nine taxa in the previous checklist, leaving it updated to 167 species, 131 genera, 30 families and nine superfamilies. For 18 already known species, new provincial data are reported expanding its known distribution within the region. The added species are presented as a commented taxonomic list and an update checklist of all the hymenopterans known until now by the region with distribution by province and ecological landscape is included.

Keywords: Hymenoptera, update, distribution, ecological landscape, Piura region, Peru.

Recibido/Received: 27/03/2021; Aceptado/Accepted: 13/10/2021; Publicado en línea/Published online: 09/12/2021

Cómo citar este artículo/Citation: Juárez-Noé, G. 2021. Actualización al listado de himenópteros (Insecta: Hymenoptera) de la región Piura, Perú. Grael/sia, 77(2): e152. https://doi.org/10.3989/graellsia.2021.v77.312

Copyright: ( 2021 SAM \& CSIC. Este es un artículo de acceso abierto distribuido bajo los términos de una licencia de uso y distribución Creative Commons Reconocimiento 4.0 Internacional (CC BY 4.0).

\section{Introducción}

Hymenoptera constituye uno de los órdenes de insectos más diversos del planeta con más de 160.000 especies y posiblemente con miles de especies aún no descritas (Sharkey, 2007; Fernández \& Pujade-Villar, 2015; Peters et al., 2017). Las especies de este orden presentan una gran variedad de formas y tamaños ocupando la mayoría de los ecosistemas, siendo algunos de importancia económica actuando como plagas forestales y otras de importancia ecológica participando en el control de plagas y en el proceso de polinización (Fernández y Pujade-Villar, 2015).

Perú no cuenta con datos exhaustivos acerca de la cantidad de especies de himenópteros que posee, sin embargo, existen trabajos que detallan la riqueza y distribución de especies para algunos grupos presentes en el país. Por ejemplo: Apidae con 14 especies dentro 
del género Bombus y 16 especies dentro de la tribu Meliponini (Rasmussen, 2003; Castillo-Carrillo et al., 2016), Aculeata con 1.196 especies (Rasmussen \& Asenjo, 2009; dos Santos et al., 2015), Braconidae con 178 especies (Redolfi de Huiza, 1994; Yu et al., 2016), Ichneumonidae con 391 especies (Carrasco, 1972; Rodríguez-Berrío et al., 2009) y Formicidae con 679 species (Escalante, 1991; Bezděčková et al., 2015; Guénard \& Economo, 2015).

La gran variedad fisiográfica y climática que caracteriza a la región Piura han determinado la existencia de zonas geomorfológicas y ecológicas importantes, originando una gran heterogeneidad de ecosistemas, hábitats y variados gradientes altitudinales dando lugar a una notable riqueza florística y faunística con altos niveles de endemismos (More et al., 2014). Para la región Piura, Juárez-Noé \& González-Coronado (2018) elaboraron un listado de himenópteros en la que registraron 146 especies, 113 géneros y 26 familias, siendo hasta el momento la única lista que detalla la cantidad y distribución de las especies del orden Hymenoptera para esta región. Por ello, en la presente investigación con la adición de 22 especies y además con la completa identificación hasta el nivel de especie de nueve taxones citados en el listado previo, actualizamos el número y distribución de las especies de himenópteros presentes en la región Piura.

\section{Material y métodos}

Área de estudio. La región Piura se ubica en el extremo noroeste de Perú $\left(04^{\circ} 59^{\prime} \mathrm{S}-80^{\circ} 25^{\prime} \mathrm{O}\right)$ con una superficie aproximada de $35.892,49 \mathrm{~km}^{2}$ y está dividida en ocho provincias políticas: Piura, Sechura, Sullana, Talara, Paita, Morropón, Huancabamba y Ayabaca. Cuenta con clima desértico y semidesértico en la costa con temperaturas máximas de $39{ }^{\circ} \mathrm{C}$ y mínimas de $18{ }^{\circ} \mathrm{C}$ y un clima húmedo y frío en la sierra con máximas de $15{ }^{\circ} \mathrm{C}$ y mínimas de $8{ }^{\circ} \mathrm{C}$, siendo las precipitaciones generalmente estacionales (diciembre a abril). Posee dos cadenas montañosas, la Cordillera de Los Amotapes (hasta los $1.600 \mathrm{msnm}$ ) y la Cordillera de los Andes (hasta casi los $4.000 \mathrm{msnm}$ ), que configuran el relieve, paisaje y contraste altitudinal de la región. Ecológicamente está conformado en su mayoría por bosques estacionalmente secos y desierto costero y en menor proporción por manglares, bosques secos interandinos, matorral montano, bosques húmedos de montaña (bosques de neblina y bosques montanos), páramo y meseta andina (More et al., 2014; MINAM, 2018).

Para los registros a través de colectas se utilizaron cinco técnicas de muestreo: (1) una trampa de intercepción, formada de malla de nailon color negro de $2.5 \mathrm{~m}$ de ancho $\times 1.5 \mathrm{~m}$ de alto sobre la cual se colocó un techo plástico transparente y a nivel del suelo una hilera de diez bandejas plásticas amarillas uno al lado del otro sin dejar espacio, llenadas hasta su mitad con agua y detergente (García, 2003). (2) una trampa Malaise tipo Townes, de malla de poliéster de color negro en la parte inferior y blanca en la parte superior, con área de intercepción de $1.65 \mathrm{~m} \times 1.10 \mathrm{~m}$, altura de $1.90 \mathrm{~m}$ y un recipiente colector de $750 \mathrm{ml}$ llenado hasta la mitad con alcohol etílico al $75 \%$. (3) trampas pitfall formadas por vasos plásticos transparentes de $500 \mathrm{ml} \mathrm{y} 9 \mathrm{~cm}$ de diámetro de abertura llenados hasta su mitad con una mezcla de siete partes de agua por una de formol al $40 \%$ y un poco de detergente (Giraldo y Arellano, 2003). Las trampas de intercepción como la trampa Malaise estuvieron activas 24 horas y revisadas cada 12 horas, mientras que las trampas pitfall se instalaron en un transecto lineal de $100 \mathrm{~m}$ con 10 trampas separadas cada $10 \mathrm{~m}$ y revisadas cada 12 horas (Juárez-Noé \& GonzálezCoronado, 2018). Además, sobre transectos de forma y longitud no definida se realizaron (4) barridos sobre la vegetación (seis pases de red) utilizando una red entomológica formada por vara rígida de $1.20 \mathrm{~m}$ de largo unida a un anillo metálico de $30 \mathrm{~cm}$ de diámetro y un tul de forma cónica de $90 \mathrm{~cm}$ de largo y (5) recolecta directa revisando principalmente hojas, tallos y flores de plantas, hojarasca, suelo, troncos podridos y bajo piedras (Sarmiento, 2006).

Las colectas, que fueron realizadas por los autores del manuscrito, se realizaron en 41 localidades de muestreo que abarcaron las ocho provincias de la región en un rango altitudinal desde el nivel del mar hasta los $3.100 \mathrm{msnm}$ (Apéndice 1), tuvieron una duración de dos días por localidad y se llevaron a cabo mensualmente desde enero de 2019 hasta el marzo de 2020.

Los registros a través de bibliografía provienen de las publicadas en revistas científicas, tesis de maestría y doctorado, siendo consultados los trabajos de Carrasco (1972), Herrera \& Álvarez (1979), Porter (1985), Mujica \& Kroschell (2011) y Moure \& Dal Molin (2012). Adicionalmente, se revisó material en la colección Gino Juárez Noé (GJN) Piura-Perú, donde se encuentran depositados los ejemplares citados por Juárez-Noé \& González-Coronado (2018) en la lista inicial de himenópteros de la región Piura, la cual permitió completar la identificación hasta el nivel de especie de nueve géneros que carecían de su epíteto especifico y que estaban citados solamente hasta el nivel de género: Brachymeria Westwood, 1829, Telenomus Hiladay, 1833, Chrysocharis Förster, 1856, Dibrachys Förster, 1856, Cotesia Cameron, 1891, Enicospilus Stephens, 1835, Amitus Haldeman, 1850, Encarsia Förster, 1878 y Acromyrmex Mayr, 1865.

Para la identificación de los especímenes colectados y de los ejemplares depositados en la colección GJN se utilizaron los trabajos de Pardo (1964), Masner (1976), Grissell \& Goodpasture (1981), Hansson (1995), Vieira-Zanella (2002), Roques \& Skrzypcznska (2003), Velásquez de Ríos \& Terán 
(2003), Myartseva \& Evans (2007), Myartseva et al. (2010), Peters \& Baur (2011), Rodrigues-Lima et al. (2012), Delvare \& Huchet (2017), Rasmussen \& González (2017), Williams et al. (2017), Fujie et al. (2018) y Bartholomay et al. (2019). Los ejemplares examinados fueron depositados en la colección del Museo de Entomología de la Universidad Nacional de Tumbes (MEUNT) y en la colección privada Gino Juárez Noé (GJN) en Piura, Perú.

Las especies añadidas en esta actualización se presentan a modo de listado taxonómico (orden, superfamilia, familia, subfamilia, género, especie) siguiendo la clasificación de Sharkey (2007), aunque estudios filogenéticos recientes (Peters et al. 2017) demuestran claramente la ausencia de monofilia en ciertos linajes y subrayan la necesidad de establecer en el futuro una nueva clasificación, en la línea sugerida por estos últimos autores. Para cada especie se incluye un breve comentario, el material examinado (país, número de ejemplares hembra y macho, provincia, distrito, zona de muestreo, coordenadas geográficas, altitud en msnm, fecha de captura, tipo de captura, colector, tipo de hábitat y código de registro cuando procedió de material depositado en la colección GJN), asimismo de una referencia para indicar al autor o autores cuando procedió de registros bibliográficos. Los nuevos taxones citados se indican con una cruz $(+)$. Además, se aporta una lista actualizada de todos los himenópteros conocidos hasta el momento para la región Piura incluyendo para cada especie su distribución por provincia y paisaje ecológico.

\section{Resultados y discusión}

En total han sido consideradas 49 especies para esta actualización, de las cuales 27 provienen de recolectas (de un total de 423 ejemplares recuperados con los métodos de colecta utilizados), 13 de registros bibliográficos y nueve de revisión de material depositado en la colección GJN. De las provenientes a través de recolectas y citas bibliográficas 22 especies, 18 géneros y cuatro familias no estaban registradas en el listado inicial de himenópteros para la región Piura (Juárez-Noé \& González-Coronado, 2018). En este listado inicial se registraron 26 familias, 113 géneros y 146 especies de los cuales 33 taxones carecían de su epíteto específico y designados como sp (Juárez-Noé \& González-Coronado, 2018). Es importante mencionar que de la lista inicial se está excluyendo a Dasymutilla homochroma Suárez, 1970 considerada recientemente como sinónimo de Dasymutilla peruviana Suárez, 1970 (Bartholomay et al., 2019). Por lo tanto, la nueva lista de himenópteros para la región Piura queda conformada por nueve superfamilias, 30 familias, 131 géneros y 167 especies, de las cuales 138 están acompañadas de su epíteto específico y 29 designadas como sp (Tabla 1).
Las familias añadidas a la lista son Encyrtidae, Figitidae, Chrysididae y Torymidae. El total de familias registradas representa ahora el $33 \%$ de las citadas a nivel mundial (Sharkey, 2007) y el $38 \%$ de las citadas a nivel neotropical (Fernández \& Sharkey, 2006). Las familias Vespidae con 25, Apidae con 23, Formicidae con 22, Crabronidae y Braconidae con 14 cada una son las que presentan mayor cantidad de especies, destacando asimismo los géneros Bombus Latreille, 1802 y Pepsis Fabricius, 1804 con seis especies cada una, mientras que 23 especies son conocidas solamente de Perú (Carrasco, 1972; Porter, 1985; Redolfi de Huiza, 1994; Urban \& Moure, 2001; Urban, 2003; Rasmussen \& Asenjo, 2009; Moure \& Dal Molin, 2012; Bezděčková et al., 2015) (Tabla 1).

Del mismo modo, para 18 especies provenientes de colectas y que se encuentran citadas en la lista inicial de himenópteros (Juárez-Noé \& González-Coronado, 2018), se añaden nuevos datos provinciales ampliando su área de distribución conocida dentro de la región. Así, Trichogramma (Trichogramma) pretiosum Riley, 1879 y Trichogramma (Trichogramma) exiguum Pinto \& Platner, 1978 se distribuyen ahora en todas las provincias y paisajes ecológicos, mientras que Aphytis chrysomphali Mercet, 1912, Microchelonus townsendi (Viereck, 1912), Triaspis vestiticida Viereck, 1912, Protapanteles muesebecki (Blanchard, 1947), Aleiodes gossypii (Muesebeck, 1960), Centris (Penthemisia) mixta Friese, 1904, Centris (Centris) maculifrons Smith, 1854, Florilegus (Florilegus) purpurascens Cockerell, 1914, Nannotrigona mellaria (Smith, 1862), Caupolicana piurensis Cockerell, 1911, Trichostictia brunneri J. Parker, 1929, Dasymutilla blattoserica (Kohl, 1882), Traumatomutilla vitelligera (Gerstäcker, 1874) y Pseudomethoca piura (Casal, 1970) se distribuyen ahora en más de una provincia pero muestran distribución concordante con el paisaje ecológico con el cual estaban citados en la lista inicial, que corresponde a bosques estacionalmente secos. Una situación similar se presenta para Eciton sin identificar sp 1 y Acromyrmex subterraneus peruanus Borgmeier, 1940 que se distribuyen en más de una provincia pero que ecológicamente se limitan a bosques secos interandinos y bosques húmedos de montaña.

Es importante mencionar que algunas de las especies registradas presentan gran interés para la región, especialmente desde el punto de vista ecológico y económico, tal es el caso de las familias Platygastridae, Figitidae, Aphelinidae, Encyrtidae, Eulophidae, Braconidae, Ichneumonidae y Trichogrammatidae cuyas especies son muy utilizadas para el control biológico de especies insectos plagas en diversos cultivos de la región Piura (Beingolea, 1959; Herrera \& Álvarez, 1979; Redolfi de Huiza, 1994; Whu \& Valdivieso, 1999; Mujica \& Kroschel, 2011; Supanta-Huarcaya, 2017; Astola-Mariscal \& NarreaCango, 2019), mientras que algunas especies de las familias Apidae, Megachilidae y Vespidae participan 
Tabla 1.- Lista actualizada de los himenópteros presentes en la región Piura, Perú. Se incluye distribución por provincias (PIU: Piura; SULL: Sullana; TAL: Talara; PAI: Paita; SECH: Sechura; MORR: Morropón; HUA: Huancabamba; AYA: Ayabaca) y paisaje ecológico (BESII: Bosque estacionalmente seco de llanura, 0-500 msnm; BESc: Bosque estacionalmente seco de colina, $500-$ 1.000 msnm; BESm: Bosque estacionalmente seco de montaña, 1.000-1.500 msnm; BSI: Bosque seco interandino, $1.500-$ 2.000 msnm; DES: Desierto, nivel del mar; MAN: Manglar, nivel del mar; BHM: Bosque húmedo de montaña, 2.000-3.100 msnm) (Juárez-Noé \& González-Coronado, 2018). Los asteriscos ${ }^{*}$ ) representan especies conocidas únicamente de Perú.

Table 1.- Update checklist of himenopterans present in the Piura region, Peru. It includes distribution by provinces (PIU: Piura; SULL: Sullana; TAL: Talara; PAI: Paita; SECH: Sechura; MORR: Morropón; HUA: Huancabamba; AYA: Ayabaca) and ecological landscape (BESII: Seasonally dry plain forest, 0-500 masl; BESc: Seasonally dry hill forest, 500-1.000 masl; BESm: Seasonally dry mountain forest, 1.000-1.500 masl; BSI: Dry inter-Andean forest, 1.500-2.000 masl; DES: Desert, sea level; MAN: mangrove swamp, sea level; BHM: mountain rainforest, 2.000-3.100 masl) (Juárez-Noé \& González-Coronado, 2018). Asterisks (*) represent species known only from Peru.

\begin{tabular}{|c|c|c|}
\hline Taxones & $\begin{array}{c}\text { Distribución } \\
\text { (provincia) (paisaje ecológico) }\end{array}$ & Referencia \\
\hline \multicolumn{3}{|l|}{$\begin{array}{l}\text { Superfamilia Evanioidea Latreille, } 1802 \\
\text { Evaniidae Leach, } 1815 \\
\text { Evania Bradley, } 1908\end{array}$} \\
\hline 1. Evania sp & $\begin{array}{l}\text { (PIU, SULL, TAL, PAI, SECH, MORR, HUA, } \\
\text { AYA) (DES, BESII, BESc, BESm, BSI, BHM) }\end{array}$ & Juárez-Noé \& González-Coronado (2018) \\
\hline $\begin{array}{l}\text { Superfamilia Platygastroidea Haliday, } \\
1833 \\
\text { Platygastridae Haliday, } 1833 \\
\text { Sceliotrachelinae Brues, } 1908\end{array}$ & & \\
\hline $\begin{array}{l}\text { Amitus Haldeman, } 1850 \\
\text { 2. Amitus spiniferus (Bréthes, 1914) } \\
\text { Telenominae Thomson, } 1860 \\
\text { Telenomus Haliday, } 1833\end{array}$ & $(\mathrm{PIU}, \mathrm{SULL})(\mathrm{BESII})$ & \\
\hline $\begin{array}{l}\text { 3. Telenomus alecto Crawford, } 1914 \\
\text { Superfamilia Cynipoidea } \\
\text { Figitidae } \\
\text { Eucoilinae }\end{array}$ & $(P I U, S U L L)(B E S I I)$ & \\
\hline $\begin{array}{l}\text { Ganaspidium Weld, } 1952 \\
\text { 4. Ganaspidium sp } \\
\text { Chalcidoidea Latreille, } 1817 \\
\text { Aphelinidae Thomson, } 1876 \\
\text { Coccophaginae Förster, } 1878 \\
\text { Encarsia Förster, } 1878\end{array}$ & (PIU) (BESII) & Mujica \& Kroschel (2011) \\
\hline 5. Encarsia tabacivora Viggiani, 1895 & $(\mathrm{PIU})(\mathrm{BESII})$ & \\
\hline 6. Encarsia formosa Gahan, 1924 & (PIU, SULL) (BESII) & \\
\hline \multicolumn{3}{|l|}{ Aphelininae Thomson } \\
\hline \multicolumn{3}{|l|}{ Aphytis Howard, 1900} \\
\hline \multicolumn{3}{|l|}{ Eretmocerinae } \\
\hline \multicolumn{3}{|l|}{ Eretmocerus } \\
\hline \multicolumn{3}{|l|}{ Zolnerowich, 1997} \\
\hline \multicolumn{3}{|l|}{ Chalcididae Latreille, 1817} \\
\hline \multicolumn{3}{|l|}{ Chalcidinae } \\
\hline $\begin{array}{l}\text { 10. Brachymeria podagrica (Fabricius, } \\
\text { 1787) }\end{array}$ & $\begin{array}{l}\text { (PIU, SULL, PAI, TAL, SECH, MORR, HUA, } \\
\text { AYA) (BESII, BESc, BESm, BSI) }\end{array}$ & \\
\hline $\begin{array}{l}\text { Encyrtidae Walker, } 1837 \\
\text { Ooencyrtus Ashmead, } 1990\end{array}$ & & \\
\hline $\begin{array}{l}\text { 11. Ooencyrtus bucculatrix (Howard, } \\
\text { 1883) }\end{array}$ & (PIU) (BESII) & Herrera \& Álvarez (1979) \\
\hline
\end{tabular}




\begin{tabular}{|c|c|c|}
\hline Taxones & $\begin{array}{c}\text { Distribución } \\
\text { (provincia) (paisaje ecológico) }\end{array}$ & Referencia \\
\hline \multicolumn{3}{|l|}{ Eulophidae Westwood, 1829} \\
\hline $\begin{array}{l}\text { 12. Chrysocharis vonones (Walker, } \\
\text { 1839) }\end{array}$ & (PIU, SULL, TAL, PAI, SECH) (BESII) & Mujica \& Kroschel (2011) \\
\hline \multicolumn{3}{|l|}{ Proacrias Ihering, 1914} \\
\hline $\begin{array}{l}\text { 13. Proacrias thysanoides (De Santis, } \\
\text { 1972) } \\
\text { Eulophinae } \\
\text { Zagrammosoma Ashmead, } 1904\end{array}$ & (PIU) (BESII) & Herrera \& Álvarez (1979) \\
\hline $\begin{array}{l}\text { 14. Zagrammosoma variegatum (Masi, } \\
\text { 1907) }\end{array}$ & 1907) & Mujica \& Kroschel (2011) \\
\hline \multicolumn{3}{|l|}{ Diglyphus } \\
\hline $\begin{array}{l}\text { 15. Diglyphus websteri (Crawford, } \\
\text { 1912) }\end{array}$ & $(\mathrm{PIU})(\mathrm{BESII})$ & Mujica \& Kroschel (2011) \\
\hline \multicolumn{3}{|l|}{ Diaulinopsis Crawford, 1912} \\
\hline $\begin{array}{l}\text { 16. Diaulinopsis callichroma Crawford, } \\
1912\end{array}$ & (PIU) (BESII) & Juárez-Noé \& González-Coronado (2018) \\
\hline \multicolumn{3}{|l|}{ Eupelmidae Walker, 1833} \\
\hline \multicolumn{3}{|l|}{$\begin{array}{l}\text { Eupelminae Walker, } 1833 \\
\text { Eupelmus Dalman, } 1820\end{array}$} \\
\hline $\begin{array}{l}\text { 17. Eupelmus (Eupelmus) pulchriceps } \\
\text { (Cameron, 1904) }\end{array}$ & (PIU, SULL) (BESII) & Juárez-Noé \& González-Coronado (2018) \\
\hline \multicolumn{3}{|l|}{ Brasema Cameron, 1884} \\
\hline $\begin{array}{l}\text { 18. Brasema peruviana (Crawford, } \\
\text { 1912) }\end{array}$ & (PIU, SULL) (BESII) & Juárez-Noé \& González-Coronado (2018) \\
\hline \multicolumn{3}{|l|}{ Eurytomidae Walker, 1832} \\
\hline \multicolumn{3}{|l|}{ Eurytominae Walker, 1832} \\
\hline $\begin{array}{l}\text { Eurytoma Illiger, } 1807 \\
\text { 19. Eurytoma piurae Crawford, } 1912\end{array}$ & (PIU) (BESII) & Juárez-Noé \& González-Coronado (2018) \\
\hline \multicolumn{3}{|l|}{ Pteromalidae Dalman, 1820} \\
\hline \multicolumn{3}{|l|}{$\begin{array}{l}\text { Pteromalinae Dalman, } 1820 \\
\text { Dibrachys Förster, } 1856\end{array}$} \\
\hline $\begin{array}{l}\text { 20. Dibrachys microgastri (Bouché, } \\
\text { 1834) }\end{array}$ & \multicolumn{2}{|c|}{ 1834) } \\
\hline \multicolumn{3}{|l|}{ Jaliscoa Bouček, 1993} \\
\hline \multirow{2}{*}{\multicolumn{3}{|c|}{$\begin{array}{l}\text { Subfamilia Miscogastrinae } \\
\text { Halticoptera }\end{array}$}} \\
\hline & & \\
\hline \multirow{2}{*}{\multicolumn{3}{|c|}{$\begin{array}{l}\text { 22. Halticoptera arduine (Walker, 1843) } \\
\text { Familia Torymidae }\end{array}$}} \\
\hline & & \\
\hline \multicolumn{3}{|l|}{$\begin{array}{l}\text { 23. Podagrion brasiliense Howard, } \\
1894\end{array}$} \\
\hline \multicolumn{3}{|l|}{ Megastigmus Dalman, 1820} \\
\hline $\begin{array}{l}\text { 24. Megastigmus transvaalensis } \\
\text { (Hussey, 1956) }\end{array}$ & (PIU, SULL, TAL, PAI, SECH) (BESII) & \\
\hline $\begin{array}{l}\text { Trichogrammatidae Haliday, } 1851 \\
\text { Trichogramma Westwood }\end{array}$ & & \\
\hline 25. Trichogramma (Trichogramma) & (PIU, SULL, TAL, PAI, SECH, MORR, HUA, & \\
\hline pretiosum Riley, 1879 & AYA) (BESII, BESc, BSI, BHM) & \\
\hline 26. Trichogramma (Trichogramma) & (PIU, SULL, TAL, PAI, SECH, MORR, HUA, & \\
\hline exiguum Pinto \& Platner, 1978 & AYA) (BESII, BESc, BSI, BHM) & \\
\hline
\end{tabular}




\begin{tabular}{|c|c|c|}
\hline Taxones & $\begin{array}{c}\text { Distribución } \\
\text { (provincia) (paisaje ecológico) }\end{array}$ & Referencia \\
\hline \multirow{5}{*}{\multicolumn{3}{|c|}{$\begin{array}{l}\text { Superfamilia Ichneumonoidea Latreille, } \\
1802 \\
\text { Ichneumonoidea Latreille Braconidae } \\
\text { Nees, } 18111802 \\
\text { Microgastrinae Förster, } 1862 \\
\text { Cotesia Cameron, } 1891\end{array}$}} \\
\hline & & \\
\hline & & \\
\hline & & \\
\hline & & \\
\hline 27. Cotesia flavipes Cameron & (PIU, SULL) (BESII) & \\
\hline \multirow{2}{*}{$\begin{array}{l}\text { 28. Apanteles sp } \\
\text { Glyptapanteles Ashmead, } 1904\end{array}$} & (PUI SUU SECH) (RESU) & 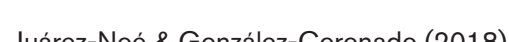 \\
\hline & (PIU, SULL, SECH) (BESII) & Juarez-INoe \& Gonzalez-Coronado (2018) \\
\hline $\begin{array}{l}\text { 29. Glyptapanteles muesebecki } \\
\text { (Blanchard, 1947) }\end{array}$ & $\begin{array}{l}\text { (PIU, SULL, TAL, PAI, SECH, MORR, AYA) } \\
\text { (BESII, BESc, BHM) }\end{array}$ & \\
\hline \multicolumn{3}{|l|}{ Cheloninae Förster, 1862} \\
\hline \multirow{2}{*}{$\begin{array}{l}\text { 30. Microchelonus townsendi (Viereck, } \\
\text { 1912) * } \\
\text { Helconinae Förster, } 1862\end{array}$} & $\begin{array}{l}\text { (PIU, SULL, TAL, PAI, SECH, MORR, AYA) } \\
\text { (BESII, BESc) }\end{array}$ & \\
\hline & & \\
\hline 31. Triaspis vestiticida Viereck, 1912 & $\begin{array}{l}\text { (PIU, SULL, PAI, TAL, MORR, SECH) (BESII, } \\
\text { BESc) }\end{array}$ & Juárez-Noé \& González-Coronado (2018) \\
\hline \multicolumn{3}{|l|}{$\begin{array}{l}\text { Aphidiinae Haliday, } 1834 \\
\text { Aphidius Nees, } 1819\end{array}$} \\
\hline \multirow{2}{*}{$\begin{array}{l}\text { 32. Aphidius sp } \\
\text { Braconinae Nees, } 1811\end{array}$} & (PIU, SULL. PAI. TAL) (BESII) & Juárez-Noé \& González-Coronado (2018) \\
\hline & & \\
\hline \multicolumn{3}{|l|}{ Habrobracon Ashmead, 1895} \\
\hline 33. Habrobracon hebetor (Say, 1836) & $\begin{array}{l}\text { (PIU, SULL, TAL, PAI, SECH, MORR, HUA, } \\
\text { AYA) (BESII, BESc, BSI, BHM) }\end{array}$ & Juárez-Noé \& González-Coronado (2018) \\
\hline \multicolumn{3}{|l|}{ Bracon Fabricius, 1804} \\
\hline 34. Bracon vestiticida (Viereck, 1912) & (PIU, SULL, TAL, PAI, SECH, MORR) (BESII) & Juárez-Noé \& González-Coronado (2018) \\
\hline \multicolumn{3}{|l|}{ Digonogastra Viereck, 1912} \\
\hline \multirow{2}{*}{\multicolumn{3}{|c|}{$\begin{array}{l}\text { 35. Digonogastra rimac (Wolcott, 1929) } \\
\text { Doryctinae Förster, } 1862\end{array}$}} \\
\hline & & \\
\hline \multicolumn{3}{|l|}{ Heterospilus Haliday, 1836} \\
\hline \multicolumn{3}{|l|}{$\begin{array}{l}\text { 36. Heterospilus hambletoni } \\
\text { Muesebeck, } 1937\end{array}$} \\
\hline \multicolumn{3}{|l|}{ Percnobracon Kieffer \& Jorgensen, } \\
\hline 37. Percnobracon secundus & (PIU, SULL, TAL, PAI, SECH) (BESII) & Juárez-Noé \& González-Coronado (2018) \\
\hline \multicolumn{3}{|l|}{ Muesebeck, 1958} \\
\hline \multicolumn{3}{|l|}{$\begin{array}{l}\text { Hormiinae Förster, } 1862 \\
\text { Cantharactonus Viereck, } 1912\end{array}$} \\
\hline \multirow{2}{*}{\multicolumn{3}{|c|}{$\begin{array}{l}1912 \\
\text { Allobracon Gahan, } 1915\end{array}$}} \\
\hline & & \\
\hline $\begin{array}{l}\text { 39. Allobracon primus (Muesebeck, } \\
\text { 1958) }\end{array}$ & (PIU, SULL, PAI) (BESII) & \\
\hline \multicolumn{3}{|l|}{$\begin{array}{l}\text { 1958) } \\
\text { Rogadinae Förster, } 1862\end{array}$} \\
\hline \multicolumn{3}{|l|}{ Aleiodes Wesmael, 1838} \\
\hline 40. Aleiodes gossypii (Muesebeck, & (PIU, SULL, PAI, TAL) (BESII) & \\
\hline \multirow{2}{*}{\multicolumn{3}{|c|}{$\begin{array}{l}\text { 1960) } \\
\text { Ichneumonidae Latreille, } 1802\end{array}$}} \\
\hline & & \\
\hline \multicolumn{3}{|l|}{ Ophioninae Shuckard, 1840} \\
\hline \multicolumn{3}{|l|}{ Enicospilus Stephens, 1835} \\
\hline 41. Enicospilus purgatus (Say, 1835) & (PIU, SULL, PAI, TAL, SECH) (BESII) & \\
\hline
\end{tabular}




\begin{tabular}{|c|c|c|}
\hline Taxones & $\begin{array}{c}\text { Distribución } \\
\text { (provincia) (paisaje ecológico) }\end{array}$ & Referencia \\
\hline $\begin{array}{l}\text { Brachycyrtus } \\
\text { 42. Brachycyrtus pretiosus Cushman, } \\
\text { 1936 } \\
\text { Isdromas }\end{array}$ & (PIU) (BESII) & Carrasco (1972) \\
\hline $\begin{array}{l}\text { 43. Isdromas peruvianus (Viereck, } \\
\text { 1912)* } \\
\text { Aeliopotes }\end{array}$ & (PIU) (BESII) & Carrasco (1972) \\
\hline $\begin{array}{l}\text { 44. Aeliopotes paitensis (Cockerell, } \\
\text { 1927)* }\end{array}$ & (PAI) (BESII) & Porter (1985) \\
\hline \multicolumn{3}{|l|}{ Cryptinae Kirby, 1837} \\
\hline $\begin{array}{l}\text { Cryptanura Brullé, } 1846 \\
\text { 45. Cryptanura sp }\end{array}$ & (HUA, AYA) (BSI, BHM) & \\
\hline \multicolumn{3}{|l|}{$\begin{array}{l}\text { 45. Cryptanura sp } \\
\text { Superfamilia Chrysidoidea }\end{array}$} \\
\hline \multicolumn{3}{|l|}{ Chrysididae Latreille, 1802} \\
\hline \multicolumn{3}{|l|}{ Chrysidinae Latreille, 1802} \\
\hline 46. Holopyga sp & (PIU) (BESII) & \\
\hline \multicolumn{3}{|l|}{ Bethylidae Haliday, 1840} \\
\hline \multicolumn{3}{|l|}{ Bethylinae Haliday, 1840} \\
\hline $\begin{array}{l}\text { Goniozus Förster, } 1856 \\
\text { 47. Goniozus sp }\end{array}$ & (PIU, SULL) (BESII) & Juárez-Noé \& González-Coronado (2018) \\
\hline \multicolumn{3}{|l|}{ Superfamilia Apoidea Latreille, 1802} \\
\hline \multicolumn{3}{|l|}{ Andrenidae Latreille, 1802} \\
\hline \multicolumn{3}{|l|}{$\begin{array}{l}\text { Panurginae Leach, } 1815 \\
\text { Callonvchium Brethes } 1922\end{array}$} \\
\hline 48. Callonychium sp & (TAL, PAI) (BESII) & Juárez-Noé \& González-Coronado (2018) \\
\hline \multicolumn{3}{|l|}{$\begin{array}{l}\text { Acamptopoeum Cockerell, } 1905 \\
\text { 49. Acamptopoeum (Acamptopoeum) }\end{array}$} \\
\hline $\begin{array}{l}\text { 49. Acamptopoeum (Acamptopoeum) } \\
\text { vagans (Cockerell, 1926)* }\end{array}$ & (PIU) (BESII) & Moure \& Dal Molin (2012) \\
\hline \multicolumn{3}{|l|}{ Apidae Latreille, 1802} \\
\hline \multirow{2}{*}{\multicolumn{3}{|c|}{$\begin{array}{l}\text { Xylocopinae Latreille, } 1802 \\
\text { Xvlocopa Latreille } 1802\end{array}$}} \\
\hline & & \\
\hline $\begin{array}{l}\text { 50. Xylocopa (Schonnherria) incarum } \\
\text { Cockerell, } 1911^{*}\end{array}$ & (PIU, SULL, PAI) (BESII, BESc, BESm, DES) & Juárez-Noé \& González-Coronado (2018) \\
\hline $\begin{array}{l}\text { 51. Xylocopa (Schonnherria) piurensis } \\
\text { Cockerell, } 1912 \\
\text { Apinae Latreille, } 1802 \\
\text { Apis Linnaeus, } 1758\end{array}$ & (PIU, SULL, PAI) (BESII, BESc, BESm, DES) & Juárez-Noé \& González-Coronado (2018) \\
\hline 52. Apis mellifera Linnaeus, 1758 & $\begin{array}{l}\text { (PIU, SULL, TAL, PAI, SECH, TAL, HUA, AYA) } \\
\text { (BESII, BESc, BESm, BSI, BHM, DES, MAN) }\end{array}$ & Juárez-Noé \& González-Coronado (2018) \\
\hline \multicolumn{3}{|l|}{ Centris Fabricius, 1804} \\
\hline $\begin{array}{l}\text { 53. Centris (Penthemisia) mixta Friese, } \\
1904\end{array}$ & $\begin{array}{l}\text { (PIU, SULL, TAL, PAI, SECH, MORR, AYA) } \\
\text { (BESII, BESc, BESm, DES, MAN) }\end{array}$ & \\
\hline $\begin{array}{l}\text { 54. Centris (Centris) maculifrons Smith, } \\
1854 \\
\text { Alloscirtetica Holmberg, } 1909\end{array}$ & $\begin{array}{l}\text { (PIU, SULL, TAL, PAI, SECH, TAL, MORR, } \\
\text { AYA) (BESII, BESc, BESm, DES, MAN) }\end{array}$ & \\
\hline $\begin{array}{l}\text { 55. Alloscirtetica sp } \\
\text { Florilegus Robertson, } 1900\end{array}$ & (PIU, SULL, TAL, PAI, SECH, TAL) (BESII, DES) & Juárez-Noé \& González-Coronado (2018) \\
\hline $\begin{array}{l}\text { 56. Florilegus (Florilegus) purpurascens } \\
\text { Cockerell, } 1914\end{array}$ & $\begin{array}{l}\text { (PIU, SULL, TAL, PAI, SECH, TAL, MORR, } \\
\text { AYA) (BESII, BESc, DES) }\end{array}$ & \\
\hline $\begin{array}{l}\text { Geotrigona Moure, } 1943 \\
\text { 57. Geotrigona fumipennis Camargo \& } \\
\text { Moure, } 1996\end{array}$ & (SULL, TAL, MORR) (BESc, BESm) & Juárez-Noé \& González-Coronado (2018) \\
\hline
\end{tabular}




\begin{tabular}{|c|c|c|}
\hline Taxones & $\begin{array}{c}\text { Distribución } \\
\text { (provincia) (paisaje ecológico) }\end{array}$ & Referencia \\
\hline \multicolumn{3}{|l|}{ Nannotrigona Cockerell, 1922} \\
\hline $\begin{array}{l}\text { 58. Nannotrigona mellaria (Smith, } \\
\text { 1862) } \\
\text { Melipona Illiger, } 1806\end{array}$ & $\begin{array}{l}\text { (PIU, SULL, TAL, PAI, SECH, MORR, AYA, } \\
\text { HUA) (BESII, BESc, BESm, DES) }\end{array}$ & \\
\hline $\begin{array}{l}\text { 59. Melipona (Michmelia) mimetica } \\
\text { Cockerell, } 1914\end{array}$ & (MORR) (BESc) & Juárez-Noé \& González-Coronado (2018) \\
\hline Trigonisca Moure, 1950 & & \\
\hline $\begin{array}{l}\text { 60. Trigonisca townsendi (Cockerell, } \\
\text { 1911) }\end{array}$ & (SULL, MORR) & Juárez-Noé \& González-Coronado (2018) \\
\hline Melitoma Lepeletier \& Serville, 1828 & & \\
\hline $\begin{array}{l}\text { 61. Melitoma segmentaria (Fabricius, } \\
\text { 1804) }\end{array}$ & (MORR) & Juárez-Noé \& González-Coronado (2018) \\
\hline \multicolumn{3}{|l|}{ Plebeia Schwartz, 1938} \\
\hline 62. Plebeia sp & $\begin{array}{l}\text { (PIU, SULL, TAL, PAI, SECH, MORR) } \\
\text { (BESII, BESC, DES) }\end{array}$ & Juárez-Noé \& González-Coronado (2018) \\
\hline \multicolumn{3}{|l|}{ Bombus Latreille, 1802} \\
\hline $\begin{array}{l}\text { 63. Bombus (Fervidobombus) } \\
\text { pauloensis Friese, } 1913\end{array}$ & (TAL, PAI) (BESII, BESc) & Juárez-Noé \& González-Coronado (2018) \\
\hline $\begin{array}{l}\text { 64. Bombus (Fervidobombus) excellens } \\
\text { Smith, } 1879 .\end{array}$ & $(\mathrm{TAL})(\mathrm{BESc})$ & Juárez-Noé \& González-Coronado (2018) \\
\hline $\begin{array}{l}\text { 65. Bombus (Funebribombus) funebris } \\
\text { Smith, } 1854\end{array}$ & (AYA, HUA) (BSI, BHM) & Juárez-Noé \& González-Coronado (2018) \\
\hline $\begin{array}{l}\text { 66. Bombus (Robustobombus) } \\
\text { ecuadorius Meunier, } 1890\end{array}$ & (HUA, AYA) (BSI, BHM) & Juárez-Noé \& González-Coronado (2018) \\
\hline $\begin{array}{l}\text { 67. Bombus (Robustobombus) } \\
\text { melaleucus Handlirsch, } 1888\end{array}$ & (HUA, AYA) (BSI, BHM) & Juárez-Noé \& González-Coronado (2018) \\
\hline $\begin{array}{l}\text { 68. Bombus (Dasybombus) handlirschi } \\
\text { Friese, } 1903 \\
\text { Santiago Urban, } 1989\end{array}$ & (TAL, MORR) (BESc) & Juárez-Noé \& González-Coronado (2018) \\
\hline $\begin{array}{l}\text { 69. Santiago wittmanni Urban, } 2003 \text { * } \\
\text { Subfamilia Nomadinae Latreille, } 1802 \\
\text { Triepeolus Robertson, } 1901\end{array}$ & $(\mathrm{HUA})(\mathrm{BHM})$ & Juárez-Noé \& González-Coronado (2018) \\
\hline $\begin{array}{l}\text { 70. Triepeolus buchwaldi (Friese, 1908) } \\
\text { Colletidae Lepeletier, } 1841\end{array}$ & (PIU, SULL) (BESII) & Juárez-Noé \& González-Coronado (2018) \\
\hline \multicolumn{3}{|l|}{ Colletinae Lepeletier, 1841} \\
\hline $\begin{array}{l}\text { 71. Colletes peruvicus Cockerell, } 1913^{*} \\
\text { Diphaglossinae Vachal, } 1909\end{array}$ & (PIU, SULL, TAL) (BESII) & Juárez-Noé \& González-Coronado (2018) \\
\hline \multicolumn{3}{|l|}{$\begin{array}{l}\text { Diphaglossinae Vachal, } 1909 \\
\text { Caupolicana Spinola. } 1851\end{array}$} \\
\hline $\begin{array}{l}\text { 72. Caupolicana piurensis Cockerell, } \\
1911^{\star} \\
\text { Ptiloglossa Smith, } 1853\end{array}$ & $\begin{array}{l}\text { (PIU, SULL, TAL, PAI, SECH, MORR) } \\
\text { (BESII, BESc) }\end{array}$ & Juárez-Noé \& González-Coronado (2018) \\
\hline $\begin{array}{l}\text { 73. Ptiloglossa ollantayi Cockerell, } \\
\text { 1911* }\end{array}$ & (PIU, SULL, TAL, PAI) (BESII) & Juárez-Noé \& González-Coronado (2018) \\
\hline $\begin{array}{l}\text { 74. Ptiloglossa magrettii (Friese, 1899) } \\
\text { Hylaeinae Viereck, } 1916\end{array}$ & $(\mathrm{HUA})(\mathrm{BSI})$ & Juárez-Noé \& González-Coronado (2018) \\
\hline Hylaeus Fabricius, 1793 & & \\
\hline 75. Hylaeus sp & (TAL) (BESII, BESc) & \\
\hline Crabronidae Latreille, 1802 & & \\
\hline Bembecinae Latreille, 1802 & & \\
\hline $\begin{array}{l}\text { Bicyrtes Lepeletier de Saint Fargeau, } \\
1845\end{array}$ & & \\
\hline
\end{tabular}




\begin{tabular}{|c|c|c|}
\hline Taxones & $\begin{array}{c}\text { Distribución } \\
\text { (provincia) (paisaje ecológico) }\end{array}$ & Referencia \\
\hline $\begin{array}{l}\text { 76. Bicyrtes variegata (Olivier, 1789) } \\
\text { Bembix Fabricius, } 1775\end{array}$ & $\begin{array}{l}\text { (PIU, SULL, TAL, PAI, SECH, MORR, AYA, } \\
\text { HUA) (BESII, BESc, DES, MAN) }\end{array}$ & Juárez-Noé \& González-Coronado (2018) \\
\hline 77. Bembix sp & $\begin{array}{l}\text { (PIU, SULL, TAL, PAI, SECH, MORR) } \\
\text { (BESII, BESc, DES, MAN) }\end{array}$ & Juárez-Noé \& González-Coronado (2018) \\
\hline $\begin{array}{l}\text { Hoplisoides Gribodo, } 1884 \\
\text { 78. Hoplisoides feae (Handlirsch, } \\
\text { 1895)* }\end{array}$ & (SULL, TAL) (BESc) & Juárez-Noé \& González-Coronado (2018) \\
\hline Rubrica J. Parker, 1929 & & \\
\hline $\begin{array}{l}\text { 79. Rubrica denticornis (Handlirsch, } \\
\text { 1890) }\end{array}$ & $\begin{array}{l}\text { (PIU, SULL, TAL, PAI, SECH, MORR, AYA) } \\
\text { (BESII, BESc, BESm, DES, MAN) }\end{array}$ & Juárez-Noé \& González-Coronado (2018) \\
\hline 80. Rubrica nasuta (Christ, 1791) & $\begin{array}{l}\text { (PIU, SULL, TAL, PAI, SECH, MORR, AYA) } \\
\text { (BESII, BESc, BESm, DES, MAN) }\end{array}$ & Juárez-Noé \& González-Coronado (2018) \\
\hline Stictia Illiger, 1807 & & \\
\hline 81. Stictia infracta J. Parker, 1929* & $\begin{array}{l}\text { (PIU, SULL, TAL, PAI, SECH, MORR, AYA) } \\
\text { (BESII, BESc, BESm, DES, MAN) }\end{array}$ & Juárez-Noé \& González-Coronado (2018) \\
\hline $\begin{array}{l}\text { Trichostictia J. Parker, } 1929 \\
\text { 82. Trichostictia brunneri J. Parker, } \\
1929\end{array}$ & (TAL, SULL) (BESc, BESm) & Juárez-Noé \& González-Coronado (2018) \\
\hline Crabroninae Latreille, 1802 & & \\
\hline $\begin{array}{l}\text { 83. Liris Fabricius, } 1804 \\
\text { Oxybelus Latreille, } 1796\end{array}$ & (PIU, SULL, PAI, TAL, SECH, MORR) (BESII) & Juárez-Noé \& González-Coronado (2018) \\
\hline $\begin{array}{l}\text { 84. Oxybelus sp } \\
\text { Trypoxylon Latreille, } 1796\end{array}$ & (PIU, SULL, TAL, SECH) (BESII, DES) & Juárez-Noé \& González-Coronado (2018) \\
\hline $\begin{array}{l}\text { 85. Trypoxylon buchwaldi Richards, } \\
1934 \\
\text { Pemphredoninae Dahlbom, } 1835 \\
\text { Incastigmus Finnamore, } 1995\end{array}$ & (PIU, SULL, PAI) (BESII) & Juárez-Noé \& González-Coronado (2018) \\
\hline $\begin{array}{l}\text { 86. Incastigmus caelukhus Finnamore, } \\
2002^{*} \\
\text { Pluto Pate, } 1937\end{array}$ & (PIU, SULL, PAI) (BESII) & Juárez-Noé \& González-Coronado (2018) \\
\hline 87. Pluto rufanalis van Lith, 1979 * & (TAL, SULL, PAI) (BESII) & Juárez-Noé \& González-Coronado (2018) \\
\hline $\begin{array}{l}\text { 88. Pluto townsendi (Cockerell, 1911) } \\
\text { Philanthinae Latreille, } 1802\end{array}$ & (PIU, SULL, TAL) (BESII) & Juárez-Noé \& González-Coronado (2018) \\
\hline Cerceris Latreille, 1802 & & \\
\hline 89. Cerceris sp & $\begin{array}{l}\text { (PIU, SULL, TAL, PAI, SECH, MORR, AYA, } \\
\text { HUA) (BESII, BESc, BESm, DES) }\end{array}$ & Juárez-Noé \& González-Coronado (2018) \\
\hline $\begin{array}{l}\text { Halictidae Thomson, } 1869 \\
\text { Halictinae Thomson, } 1869 \\
\text { Agapostemon Guérin-Méneville, } 1844\end{array}$ & \multicolumn{2}{|c|}{ Halictinae Thomson, 1869} \\
\hline $\begin{array}{l}\text { 90. Agapostemon (Notagapostemon) } \\
\text { nasutus } \\
\text { Megachilidae Latreille, } 1802 \\
\text { Megachilinae Latreille, } 1802 \\
\text { Anthidium Fabricius, } 1804\end{array}$ & $\begin{array}{l}\text { (PIU, SULL, TAL, PAI, SECH, MORR, HUA, } \\
\text { AYA) (BESII, BESc, BESm, DES) }\end{array}$ & Juárez-Noé \& González-Coronado (2018) \\
\hline 91. Anthidium paitense Cockerell, 1926 & (PAI, SULL, TAL) (BESII) & Juárez-Noé \& González-Coronado (2018) \\
\hline $\begin{array}{l}\text { 92. Anthidium vigintiduopunctatum } \\
\text { Friese, } 1904 \\
\text { Anthodioctes Holmberg, } 1903\end{array}$ & $(\mathrm{PIU}, \mathrm{SULL})(\mathrm{BESII})$ & Juárez-Noé \& González-Coronado (2018) \\
\hline 93. Anthodioctes sp & $\begin{array}{l}\text { (PIU, SULL, TAL, PAI, SECH, MORR, HUA, } \\
\text { AYA) (BESII, BESc) }\end{array}$ & Juárez-Noé \& González-Coronado (2018) \\
\hline
\end{tabular}




\begin{tabular}{|c|c|c|}
\hline Taxones & $\begin{array}{c}\text { Distribución } \\
\text { (provincia) (paisaje ecológico) }\end{array}$ & Referencia \\
\hline \multicolumn{3}{|l|}{ Megachile Latreille, 1802} \\
\hline $\begin{array}{l}\text { 94. Megachile (Pseudocentron) } \\
\text { aricensis Friese, } 1904\end{array}$ & (PIU, SULL, TAL, PAI, SECH) (BESII) & Juárez-Noé \& González-Coronado (2018) \\
\hline 95. Megachile (Austromegachile) & (PIU) (BESII) & Moure \& Dal Molin (2012) \\
\hline $\begin{array}{l}\text { philinca Cockerell, } 1912 \\
\text { 96. Megachile (Dasymegachile) }\end{array}$ & (PIU) (BESII) & Moure \& Dal Molin (2012) \\
\hline \multirow{2}{*}{\multicolumn{3}{|c|}{$\begin{array}{l}\text { piurensis Cockerell, } 1911 \text { * } \\
\text { Coelioxys Latreille, } 1809\end{array}$}} \\
\hline & & \\
\hline 97. Coelioxys sp & (PIU, SULL, PAI) (BESII) & Juárez-Noé \& González-Coronado (2018) \\
\hline \multicolumn{3}{|l|}{ Sphecidae Latreille, 1802} \\
\hline \multicolumn{3}{|l|}{ Ammophilinae André, 1886} \\
\hline \multicolumn{3}{|l|}{ Ammophila W.F. Kirby, 1798} \\
\hline 98. Ammophila sp & $\begin{array}{l}\text { (PIU, SULL, TAL, PAI, SECH, MORR, AYA, } \\
\text { HUA) (BESII, BESc, DES) }\end{array}$ & Juárez-Noé \& González-Coronado (2018) \\
\hline \multicolumn{3}{|l|}{ Sceliphrinae Ashmead, 1899} \\
\hline \multicolumn{3}{|l|}{ Chalybion Dahlbom, 1843} \\
\hline 99. Chalybion sp & $\begin{array}{l}\text { (PIU, SULL, TAL, PAI, SECH, MORR) } \\
\text { (BESII, BESc, DES) }\end{array}$ & Juárez-Noé \& González-Coronado (2018) \\
\hline \multicolumn{3}{|l|}{ Sceliphron Klug, 1801} \\
\hline $\begin{array}{l}\text { 100. Sceliphron (Sceliphron) } \\
\text { caementarium (Drury, 1773) }\end{array}$ & $\begin{array}{l}\text { (PIU, SULL, TAL, PAI, SECH, MORR, AYA, } \\
\text { HUA) (BESII, BESc, BSI, BHM, DES, MAN) }\end{array}$ & Juárez-Noé \& González-Coronado (2018) \\
\hline 101. Sceliphron (Sceliphron) fistularium & (PIU, SULL, TAL, PAI, SECH, MORR) & Juárez-Noé \& González-Coronado (2018) \\
\hline \multicolumn{3}{|l|}{ Sphecinae Latreille, 1802} \\
\hline Prionyx Vander Linden, 1827 & & Juárez-Noé \& González-Coronado (2018) \\
\hline 102. Prionyx thomae (Fabricius, 1775 ) & $\begin{array}{l}\text { PIU, SULL, TAL, PAI, SECH, MORR) } \\
\text { (BESII, BESc, DES) }\end{array}$ & \\
\hline \multicolumn{3}{|l|}{ Superfamilia Vespoidea Latreille, 1802} \\
\hline \multicolumn{3}{|l|}{$\begin{array}{l}\text { Ponerinae Lepeletier de Saint-Fargeau, } \\
1835\end{array}$} \\
\hline \multicolumn{3}{|l|}{ Odontomachus Latreille, 1804} \\
\hline 103. Odontomachus sp & $\begin{array}{l}\text { (PIU, SULL, TAL. PAI, SECH, MORR, HUA, } \\
\text { AYA) (BESIl, BESc, BESm, BSI) }\end{array}$ & Juárez-Noé \& González-Coronado (2018) \\
\hline \multicolumn{3}{|l|}{ Hypoponera (Santschi, 1938) } \\
\hline $\begin{array}{l}\text { 104. Hypoponera opaciceps (Mayr, } \\
\text { 1887) }\end{array}$ & (SULL, TAL, MORR) (BESc) & Juárez-Noé \& González-Coronado (2018) \\
\hline \multicolumn{3}{|l|}{ Neoponera Emery, 1901} \\
\hline $\begin{array}{l}\text { 105. Neoponera commutata (Roger, } \\
\text { 1860) }\end{array}$ & (MORR) (BESc) & Juárez-Noé \& González-Coronado (2018) \\
\hline \multicolumn{3}{|l|}{ Dorylinae Leach, 1815} \\
\hline \multicolumn{3}{|l|}{ Cylindromyrmex Mayr, 1870} \\
\hline $\begin{array}{l}\text { 106. Cylindromyrmex striatus Mayr, } \\
1870\end{array}$ & $\begin{array}{l}\text { (SULL, PIU, PAI, TAL, MORR, SECH) } \\
\text { (BESII, BESc) }\end{array}$ & Juárez-Noé \& González-Coronado (2018) \\
\hline \multicolumn{3}{|l|}{ Eciton Latreille, 1804} \\
\hline 107. Eciton sp & (HUA, AYA) & Juárez-Noé \& González-Coronado (2018) \\
\hline \multicolumn{3}{|l|}{ Dolichoderinae Forel, 1878} \\
\hline \multicolumn{3}{|l|}{ Dorymyrmex Mayr, 1866} \\
\hline $\begin{array}{l}\text { 108. Dorymyrmex pyramicus peruvianus } \\
\text { Wheeler W. M. } 1919^{*}\end{array}$ & $\begin{array}{l}\text { (PIU, SULL, TAL, PAI, MORR, SECH) } \\
\text { (BESII, BESc, BESm, DES, MAN) }\end{array}$ & Juárez-Noé \& González-Coronado (2018) \\
\hline \multicolumn{3}{|l|}{ Linepithema Mayr, 1866} \\
\hline 109. Linepithema humile (Mayr, 1868) & $\begin{array}{l}\text { (PIU, SULL, TAL, PAI, SECH, MORR, AYA, } \\
\text { HUA) (BESII, BESc, BESm, BSI, BHM) }\end{array}$ & Juárez-Noé \& González-Coronado (2018) \\
\hline \multicolumn{3}{|l|}{ Tapinoma Foerster, 1850} \\
\hline $\begin{array}{l}\text { 110. Tapinoma melanocephalum } \\
\text { (Fabricius, 1973) }\end{array}$ & $\begin{array}{l}\text { (PIU, SULL, TAL, PAI, SECH, MORR) (BESII, } \\
\text { BESc, BESm) }\end{array}$ & Juárez-Noé \& González-Coronado (2018) \\
\hline
\end{tabular}




\begin{tabular}{|c|c|c|}
\hline Taxones & $\begin{array}{c}\text { Distribución } \\
\text { (provincia) (paisaje ecológico) }\end{array}$ & Referencia \\
\hline \multicolumn{3}{|l|}{ Formicinae Latreille, 1809} \\
\hline 111. Camponotus sp & $\begin{array}{l}\text { (PIU, SULL, TAL, MORR, PAI, SECH) } \\
\text { (BESII, BESc, BESm, DES) }\end{array}$ & Juárez-Noé \& González-Coronado (2018) \\
\hline \multicolumn{3}{|l|}{ Paratrechina Motschoulsky, 1863} \\
\hline \multicolumn{2}{|l|}{$\begin{array}{l}\text { Myrmicinae Lepeletier de Saint-Fargeau, } \\
1835 \\
\text { Acromyrmex Mayr. } 1865\end{array}$} & \\
\hline \multicolumn{3}{|l|}{ peruvianus Borgmeier, 1940} \\
\hline \multicolumn{3}{|l|}{ Cephalotes Latreille, 1802} \\
\hline 114. Cephalotes inca (Santschi, 1911) & $\begin{array}{l}\text { (PIU, SULL, TAL, PAI, SECH, MORR, AYA, } \\
\text { HUA) (BESII, BESc, BESm, DES, MAN) }\end{array}$ & Juárez-Noé \& González-Coronado (2018) \\
\hline 115. Cephalotes sp & (PIU, SULL, TAL, PAI) (BESII) & Juárez-Noé \& González-Coronado (2018) \\
\hline \multicolumn{3}{|l|}{ Crematogaster Lund, 1831} \\
\hline 116. Crematogaster crinosa Mayr, 1862 & $\begin{array}{l}\text { (PIU, SULL, PAI, TAL, SECH, MORR, HUA, } \\
\text { AYA) (BESII, BESc, BESm, DES, MAN) }\end{array}$ & Juárez-Noé \& González-Coronado (2018) \\
\hline \multicolumn{3}{|l|}{ Pheidole Westwood, 1839} \\
\hline 117. Pheidole chilensis Mayr, 1862 & $\begin{array}{l}\text { (PIU, SULL, PAI, TAL, SECH, MORR, HUA, } \\
\text { AYA) (BESII, BESc, BESm, DES, MAN) }\end{array}$ & Juárez-Noé \& González-Coronado (2018) \\
\hline \multicolumn{3}{|l|}{ Solenopsis Westwood, 1840} \\
\hline 118. Solenopsis bruesi Creighton, 1930 & (PIU, SULL, PAI) (BESII) & Juárez-Noé \& González-Coronado (2018) \\
\hline \multicolumn{3}{|l|}{ Tetramorium Mayr, 1855} \\
\hline $\begin{array}{l}\text { 120. Tetramorium bicarinatum (Nylander, } \\
\text { 1846) } \\
\text { Wasmannia Forel, } 1893\end{array}$ & $\begin{array}{l}\text { (PIU, SULL, TAL, PAI, SECH, MORR) } \\
\text { (BESII, BESc) }\end{array}$ & Juárez-Noé \& González-Coronado (2018) \\
\hline $\begin{array}{l}\text { 121. Wasmannia auropunctata (Roger, } \\
\text { 1863) } \\
\text { Pseudomyrmecinae Smith, } 1952 \\
\text { Pseudomyrmex Lund, } 1831\end{array}$ & $\begin{array}{l}\text { (PIU, SULL, TAL, PAI, SECH, MORR) } \\
\text { (BESII, BESc) }\end{array}$ & Juárez-Noé \& González-Coronado (2018) \\
\hline $\begin{array}{l}\text { 122. Pseudomyrmex gracilis (Fabricius, } \\
\text { 1804) }\end{array}$ & $\begin{array}{l}\text { (PIU, SULL, TAL, SECH, HUA, PAI, MORR, } \\
\text { HUA) (BESII, BESc, BESm, DES, MAN) }\end{array}$ & Juárez-Noé \& González-Coronado (2018) \\
\hline $\begin{array}{l}\text { 123. Pseudomyrmex simplex (Smith, } \\
\text { 1877) }\end{array}$ & $\begin{array}{l}\text { (PIU, SULL, TAL, SECH, MORR, PAI, HUA, } \\
\text { AYA) (BESII, BESc, BESm, DES, MAN) }\end{array}$ & Juárez-Noé \& González-Coronado (2018) \\
\hline $\begin{array}{l}\text { 124. Pseudomyrmex sp } \\
\text { Mutillidae Latreille, } 1802\end{array}$ & (PIU, SULL, SECH) (BESII) & Juárez-Noé \& González-Coronado (2018) \\
\hline \multicolumn{3}{|l|}{ Sphaeropthalminae Schuster, 1949} \\
\hline \multicolumn{3}{|l|}{$\begin{array}{l}\text { Quwitilla Williams, Bartholomay \& } \\
\text { Cambra. } 2019\end{array}$} \\
\hline 125. Quwitilla blattoserica (Kohl, 1882) & $\begin{array}{l}\text { (SULL, PIU, TAL, PAI, SECH, MORR. AYA) } \\
\text { (BESII, BESc, DES) }\end{array}$ & \\
\hline $\begin{array}{l}\text { 126. Quwitilla peruviana (Suárez, 1970) } \\
\text { Traumatomutilla André, } 1901\end{array}$ & (PIU, TAL, SECH) (BESII) & Juárez-Noé \& González-Coronado (2018) \\
\hline $\begin{array}{l}\text { 127. Traumatomutilla vitelligera } \\
\text { (Gerstäcker, 1874) }\end{array}$ & $\begin{array}{l}\text { (PIU, SULL, TAL, PAI, SECH, MORR, AYA, } \\
\text { HUA) (BESII, BESc) }\end{array}$ & \\
\hline Euspinolia Ashmead, 1903 & & \\
\hline $\begin{array}{l}\text { 128. Euspinolia krombeini Casal, } 1964 \\
\text { Pseudomethoca Ashmead, } 1896\end{array}$ & $(\mathrm{SULL})(\mathrm{BESII})$ & Juárez-Noé \& González-Coronado (2018) \\
\hline $\begin{array}{l}\text { 129. Pseudomethoca piura (Casal, } \\
\text { 1970) }\end{array}$ & $\begin{array}{l}\text { (PIU, SULL, PAI, SECH, TAL, MORR) } \\
\text { (BESII, BESc) }\end{array}$ & \\
\hline
\end{tabular}




\begin{tabular}{|c|c|c|}
\hline Taxones & $\begin{array}{c}\text { Distribución } \\
\text { (provincia) (paisaje ecológico) }\end{array}$ & Referencia \\
\hline \multicolumn{3}{|l|}{ Mutillinae Latreille, 1802} \\
\hline \multicolumn{3}{|l|}{ Ephuta Say, 1836} \\
\hline 130. Ephuta inca Suárez, 1970 & $(\mathrm{SULL})(\mathrm{BESII})$ & Juárez-Noé \& González-Coronado (2018) \\
\hline Timulla Ashmead, 1899 & & \\
\hline 131. Timulla inca Mickel, 1938 & (PIU) (BESII) & Juárez-Noé \& González-Coronado (2018) \\
\hline \multicolumn{3}{|l|}{ Pompilidae Latreille, 1804} \\
\hline \multirow{2}{*}{\multicolumn{3}{|c|}{$\begin{array}{l}\text { Pepsinae Fabricius, } 1804 \\
\text { Auplopus Spinola, } 1841\end{array}$}} \\
\hline & & \\
\hline $\begin{array}{l}\text { 132. Auplopus eriodes Dreisbach, } \\
1963^{*}\end{array}$ & (PIU, SULL, TAL) (BESII, DES) & Juárez-Noé \& González-Coronado (2018) \\
\hline \multicolumn{3}{|l|}{ Pepsis Fabricius, 1804} \\
\hline 133. Pepsis grossa (Fabricius, 1798) & (PIU) (BESII) & Juárez-Noé \& González-Coronado (2018) \\
\hline $\begin{array}{l}\text { 134. Pepsis inclyta Lepeletier de Saint } \\
\text { Fargeau, } 1845\end{array}$ & (MORR, HUA, AYA) (BESm, BSI, BHM) & Juárez-Noé \& González-Coronado (2018) \\
\hline 135. Pepsis multichroma Vardy, 2005 & (PIU, SULL, TAL, PAI, SECH, HUA) (BESII, DES) & Juárez-Noé \& González-Coronado (2018) \\
\hline 136. Pepsis montezuma F. Smith, 1855 & $\begin{array}{l}\text { (PIU, SULL, TAL, MORR, SECH, HUA, AYA) } \\
\text { (BESII, BESc, DES) }\end{array}$ & Juárez-Noé \& González-Coronado (2018) \\
\hline $\begin{array}{l}\text { 137. Pepsis petitii Guérin-Méneville, } \\
1831\end{array}$ & $\begin{array}{l}\text { (PIU, SULL, SECH, TAL, HUA, AYA) } \\
\text { (BESII, BESc, BESm, DES) }\end{array}$ & Juárez-Noé \& González-Coronado (2018) \\
\hline $\begin{array}{l}\text { 138. Pepsis chiliensis Lepeletier de } \\
\text { Saint Fargeau. } 1845\end{array}$ & (PIU, TAL) (BESII, BESc) & Juárez-Noé \& González-Coronado (2018) \\
\hline \multicolumn{3}{|l|}{$\begin{array}{l}\text { Pant Fargeau, } 1845 \\
\text { Pompilinae Latreille, } 1805\end{array}$} \\
\hline \multicolumn{3}{|l|}{ Poecilopompilus Howard, 1901} \\
\hline 139. Poecilopompilus rubricatus (F. & (TAL, SECH, MORR, PAI, SULL) & Juárez-Noé \& González-Coronado (2018) \\
\hline Smith, 1879) & (BESII, BESc, BESm, DES) & \\
\hline \multicolumn{3}{|l|}{ Scoliidae Leach, 1815} \\
\hline \multicolumn{3}{|l|}{ Campsomerinae Leach, 1815} \\
\hline \multicolumn{3}{|l|}{ Campsomeris Guérin-Méneville, 1839} \\
\hline 140. Campsomeris sp & $\begin{array}{l}\text { (PIU, SULL, TAL, PAI, SECH, MORR, HUA, AYA) } \\
\text { (BESII, BESc, BESm, BSI, BHM, DES, MAN) }\end{array}$ & Juárez-Noé \& González-Coronado (2018) \\
\hline \multicolumn{3}{|l|}{ Tiphiidae Leach, 1815} \\
\hline \multicolumn{3}{|l|}{ Myzininae Leach, 1815} \\
\hline \multicolumn{3}{|l|}{ Myzinum Latreille, 1803} \\
\hline 141. Myzinum sp & $\begin{array}{l}\text { (PIU, SULL, TAL, PAI, SECH, MORR) (BESII, } \\
\text { BESc, BESm) }\end{array}$ & Juárez-Noé \& González-Coronado (2018) \\
\hline \multicolumn{3}{|l|}{ Tiphiinae Leach, 1815} \\
\hline Tiphia Fabricius, 1775 & & \\
\hline 142. Tiphia fulvitarsis Rohwer, $1912^{*}$ & (PIU, SULL, TAL) (BESII, BESc) & Juárez-Noé \& González-Coronado (2018) \\
\hline \multicolumn{3}{|l|}{ Vespidae Leach, 1815} \\
\hline \multicolumn{3}{|l|}{ Eumeninae Leach, 1815} \\
\hline \multicolumn{3}{|l|}{ Monobia de Saussure, 1852} \\
\hline $\begin{array}{l}\text { 143. Monobia cyanipennis (Guérin- } \\
\text { Méneville, 1831) }\end{array}$ & $\begin{array}{l}\text { (TAL, PAI, PIU, SULL, SECH, MORR, AYA, } \\
\text { HUA) (BESII, BESc, BESm, BSI, DES, MAN) }\end{array}$ & Juárez-Noé \& González-Coronado (2018) \\
\hline \multicolumn{3}{|l|}{ Minixi Giordani Soika, 1978} \\
\hline \multirow{2}{*}{\multicolumn{3}{|c|}{$\begin{array}{l}\text { Omicron de Saussure, } 1855 \\
\text { Omixl }\end{array}$}} \\
\hline & & \\
\hline 145. Omicron sp & (PIU, SULL, TAL, PAI) (BESII) & Juárez-Noé \& González-Coronado (2018) \\
\hline Pachodynerus de Saussure, 1870 & & \\
\hline 146. Pachodynerus diabolicus (de & (TAL, PAI, PIU, SULL, SECH) & Juárez-Noé \& González-Coronado (2018) \\
\hline Saussure, 1853$)^{\star}$ & (BESII, BESc, BESm, DES) & \\
\hline
\end{tabular}




\begin{tabular}{|c|c|c|}
\hline Taxones & $\begin{array}{c}\text { Distribución } \\
\text { (provincia) (paisaje ecológico) }\end{array}$ & Referencia \\
\hline $\begin{array}{l}\text { 147. Pachodynerus gaullei Brèthes, } \\
1920\end{array}$ & (HUA) & Juárez-Noé \& González-Coronado (2018) \\
\hline $\begin{array}{l}\text { 148. Pachodynerus peruensis (de } \\
\text { Saussure, 1855) }\end{array}$ & $\begin{array}{l}\text { (TAL, PAI, PIU, SULL, SECH) } \\
\text { (BESII, BESc, BESm, DES) }\end{array}$ & Juárez-Noé \& González-Coronado (2018) \\
\hline $\begin{array}{l}\text { Zeta de Saussure, } 1855 \\
\text { 149. Zeta argillaceum (Linnaeus, 1758) }\end{array}$ & $\begin{array}{l}\text { (PIU, SULL, TAL, PAI, SECH, MORR, AYA, HUA) } \\
\text { (BESII, BESc, BESm, BSI, BHM, DES, MAN) }\end{array}$ & Juárez-Noé \& González-Coronado (2018) \\
\hline $\begin{array}{l}\text { Zethus Fabricius, } 1804 \\
\text { 150. Zethus (Zethusculus) westwoodi } \\
\text { de Saussure, } 1852 \\
\text { Ancistroceroides Saussure, } 1855\end{array}$ & (TAL, PIU, SULL, PAI) (BESII) & Juárez-Noé \& González-Coronado (2018) \\
\hline $\begin{array}{l}\text { 151. Ancistroceroides sp } \\
\text { Parancistrocerus Bequaert, } 1925\end{array}$ & $(\mathrm{TAL})(\mathrm{BESc})$ & \\
\hline $\begin{array}{l}\text { 152. Parancistrocerus } \mathrm{sp} \\
\text { Masarinae Latreille, } 1802 \\
\text { Trimeria de Saussure, } 1854\end{array}$ & $(\mathrm{TAL})(\mathrm{BESc})$ & \\
\hline $\begin{array}{l}\text { 153. Trimeria cockerelli (Bequaert, } \\
\text { 1928)* } \\
\text { Polistinae Lepeletier, } 1836 \\
\text { Agelaia Lepeletier de Saint Fargeau, } \\
1836\end{array}$ & (PAI, PIU, TAL) (BESII) & Juárez-Noé \& González-Coronado (2018) \\
\hline $\begin{array}{l}\text { 154. Agelaia pallipes festae (Zavattari, } \\
\text { 1906) }\end{array}$ & $(\mathrm{HUA})(\mathrm{BHM})$ & Juárez-Noé \& González-Coronado (2018) \\
\hline $\begin{array}{l}\text { Brachygastra Perty, } 1833 \\
\text { 155. Brachygastra augusti (de } \\
\text { Saussure, 1854) }\end{array}$ & (SULL, AYA, PAI) (BESII) & Juárez-Noé \& González-Coronado (2018) \\
\hline $\begin{array}{l}\text { 156. Brachygastra lecheguana (Latreille, } \\
\text { 1824) }\end{array}$ & $\begin{array}{l}\text { (SULL, MORR, AYA, PIU, PAI, TAL, SECH, } \\
\text { AYA, HUA) (BESII, BESc, BESm, DES, MAN) }\end{array}$ & Juárez-Noé \& González-Coronado (2018) \\
\hline $\begin{array}{l}\text { 157. Brachygastra baccalaurea (R. von } \\
\text { lhering, 1903) }\end{array}$ & (HUA, AYA) (BSI, BHM) & Juárez-Noé \& González-Coronado (2018) \\
\hline $\begin{array}{l}\text { Parachartergus R. von Ihering, } 1904 \\
\text { 158. Parachartergus colobopterus } \\
\text { (Liechtenstein, 1796) }\end{array}$ & (PIU) (BESII) & Juárez-Noé \& González-Coronado (2018) \\
\hline $\begin{array}{l}\text { 159. Parachartergus smithii (de } \\
\text { Saussure, 1854) } \\
\text { Polybia Lepeletier de Saint Fargeau, } \\
1836\end{array}$ & (MORR, AYA, HUA) (BESm, BSI) & Juárez-Noé \& González-Coronado (2018) \\
\hline $\begin{array}{l}\text { 160. Polybia (Myrapetra) fastidiosuscula } \\
\text { de Saussure, } 1854\end{array}$ & (PIU) (BESII) & Juárez-Noé \& González-Coronado (2018) \\
\hline $\begin{array}{l}\text { 161. Polybia (Myrapetra) flavifrons } \\
\text { barbatula Richards, } 1978\end{array}$ & (MORR, PIU, SULL, AYA) & Juárez-Noé \& González-Coronado (2018) \\
\hline $\begin{array}{l}\text { 162. Polybia synoecoides ignobilis } \\
\text { (Haliday, 1836) } \\
\text { Synoeca de Saussure, } 1852\end{array}$ & (MORR, PIU, SULL, PAI) (BES॥, BESc) & Juárez-Noé \& González-Coronado (2018) \\
\hline $\begin{array}{l}\text { 163. Synoeca septentrionalis Richards, } \\
1978\end{array}$ & (SULL, AYA, PAI) (BESII) & Juárez-Noé \& González-Coronado (2018) \\
\hline $\begin{array}{l}\text { Mischocyttarus de Saussure, } 1853 \\
\text { 164. Mischocyttarus (Omega) petiolatus } \\
\text { Zikán, } 1949 \\
\text { Polistes Latreille, } 1802\end{array}$ & (PIU) (BESII) & Juárez-Noé \& González-Coronado (2018) \\
\hline $\begin{array}{l}\text { 165. Polistes (Aphanilopterus) } \\
\text { peruvianus Bequaert, } 1934^{*}\end{array}$ & (PIU) (BESII) & Juárez-Noé \& González-Coronado (2018) \\
\hline $\begin{array}{l}\text { 166. Polistes (Aphanilopterus) versicolor } \\
\text { versicolor (Olivier, 1791) }\end{array}$ & $\begin{array}{l}\text { (MORR, PIU, SULL, TAL, PAI, SECH) } \\
\text { (BESII, BESc, BESM, DES) }\end{array}$ & Juárez-Noé \& González-Coronado (2018) \\
\hline $\begin{array}{l}\text { 167. Polistes (Aphanilopterus) } \\
\text { weyrauchorum Willink, } 1964\end{array}$ & $\begin{array}{l}\text { (PAI, PIU, TAL, SECH, SULL) (BES॥, BESc, } \\
\text { BESM, DES) }\end{array}$ & Juárez-Noé \& González-Coronado (2018) \\
\hline
\end{tabular}


en el proceso de polinización en algunas especies vegetales especialmente de aquellas que habitan en los ecosistemas secos de la región (Juárez-Noé \& González-Coronado, 2020).

En cuanto a los métodos de captura, 13 especies se recogieron a través de barridos de vegetación, siete especies mediante trampas Malaise y trampas de intercepción, seis especies a través de colecta manual y cinco especies con trampas pitfall. Eciton sin identificar sp. 1., A. subterraneus peruanus, $D$. blattoserica, $T$. vitelligera y $P$. piura fueron capturados mediante trampas pitfall, método ideal para la captura de himenópteros que frecuentan la superficie y hojarasca, sin embargo, estas mismas especies también fueron capturadas mediante recolecta directa sobre la superficie, incluso en áreas despejadas de bosque desprovistas de vegetación. Del mismo modo, $N$. mellaria, C. piurensis, T. brunneri, M. townsendi, T. vestiticida, P. muesebecki y A. gossypii fueron capturados simultáneamente mediante trampas de intercepción, trampas Malaise y barridos de la vegetación, mientras que Ancistroceroides sin identificar sp. 1., Parancistrocerus sin identificar sp. 1., Holopyga sin identificar sp. 1., C. mixta, C. maculifrons, $F$. purpurascens, Encarsia formosa Gahan, 1924, Encarsia pergandiella Foerster, 1878, Eretmocerus eremicus (Rose \& Zolnerowich, 1997), Cryptanura sin identificar sp. 1. y Megastigmus transvaalensis (Hussey, 1956) fueron capturados exclusivamente mediante barridos de vegetación у $T$. pretiosum, $T$. exiguum, $A$. chrysomphali y Podagrion brasiliense Howard, 1894 se capturaron exclusivamente con trampas malaise.

Por último, sería recomendable seguir completando la identificación de aquellos especímenes que quedaron identificados solo hasta el nivel de género y realizar colectas de forma continuada especialmente en áreas aún no exploradas en la región con lo que probablemente continúe elevándose el número de especies de himenópteros para la región Piura. Sin embargo, dada la alta diversidad de Hymenoptera en el mundo, debe de considerarse este listado aún básico y preliminar.

\section{Listado faunístico}

Superfamilia Platygastroidea Haliday, 1833

Familia Platygastridae Haliday, 1833

Subfamilia Sceliotrachelinae Brues, 1908

Género Amitus Haldeman, 1850

1. Amitus spiniferus (Bréthes, 1914)

Material eXAminado.PERÚ. 1 đo, Piura, Castilla, caserío Miraflores, $05^{\circ} 16^{\prime} 66^{\prime \prime} \mathrm{S}-80^{\circ} 61^{\prime} 67^{\prime \prime} \mathrm{O}, 29 \mathrm{msnm}, 09-\mathrm{II}-2018$, trampa Malaise, G. Juárez, Bosque estacionalmente seco de llanura, PLA002GJN; 1 ô, Piura, Tambogrande, $04^{\circ} 55^{\prime} 38^{\prime \prime} \mathrm{S}-80^{\circ} 21^{\prime} 09^{\prime \prime} \mathrm{O}$, 25 msnm, 06-III-2017, trampa Malaise, G. Juárez, Bosque estacionalmente seco de llanura, PLA004-GJN; 1 ㅇ, Sullana, Querecotillo, 04 ${ }^{\circ} 50^{\prime} 24^{\prime \prime} \mathrm{S}-80^{\circ} 38^{\prime} 57^{\prime \prime} \mathrm{O}, 32 \mathrm{msnm}, 24-\mathrm{VI}-2016$, red entomológica, G. Juárez, Bosque estacionalmente seco de llanura, PLA007-GJN.
Comentario. Mencionada como Amitus sp en Juárez-Noé \& González-Coronado (2018). Especie originaria de América Central, introducido en países de América del Sur y Europa (Myartseva et al., 2012). Es un endoparasitoide de huevos y ninfas de especies de Aleyrodidae (Hemiptera), especialmente de Aleurothrixus floccosus Maskell, 1896 "mosquita blanca plaga de los cítricos" (Beingolea, 1959). Los individuos examinados fueron colectados en cultivos de limón.

Subfamilia Telenominae Thomson, 1860

Género Telenomus Haliday, 1833

2. Telenomus alecto Crawford, 1914

Material examinado. PERÚ. 1 o, Piura, Castilla, Universidad NacionaldePiura, $05^{\circ} 10^{\prime} 51^{\prime \prime} \mathrm{S}-80^{\circ} 37^{\prime} 31^{\prime \prime} \mathrm{O}, 29 \mathrm{msnm}, 2-\mathrm{II}-2017$, red entomológica, G. Juárez, Bosque estacionalmente seco de llanura, PLA011-GJN; 1 Oे, Piura, Castilla, caserío Miraflores, $05^{\circ} 16^{\prime} 66^{\prime \prime} \mathrm{S}-80^{\circ} 61^{\prime} 67^{\prime \prime} \mathrm{O}, 29 \mathrm{msnm}, \quad 09-\mathrm{II}-2018$, trampa malaise, G. Juárez, Bosque estacionalmente seco de llanura, PLA014-GJN; 1 ㅇ, Sullana, Salitral, 04 $51^{\circ} 27^{\prime \prime} \mathrm{S}-80^{\circ} 40^{\prime} 52^{\prime \prime} \mathrm{O}$, 32 msnm, 16-IX-2017, red entomológica, G. Juárez, Bosque estacionalmente seco de llanura, PLA019-GJN; 1 , Sullana, Querecotillo, 04 ${ }^{\circ} 50^{\prime} 24^{\prime \prime} \mathrm{S}-80^{\circ} 38^{\prime} 57^{\prime \prime} \mathrm{O}, 32 \mathrm{msnm}, 24-\mathrm{VI}-2016$, red entomológica, G. Juárez, Bosque estacionalmente seco de llanura, PLA020-GJN.

Comentario. Mencionada como Telenomus sp en Juárez-Noé \& González-Coronado (2018). Especie originaria de Asia que es parasitoide de huevos en especies de Hemiptera y Lepidoptera (da Silva Dias et al., 2011). En Perú se utiliza para el control biológico de especies de Spodoptera Guenée, 1852 (Noctuidae) en cultivo de maíz y de especies de Diatraea (Crambidae) en cultivo de caña de azúcar (Cueva, 1980; Gómez de Picho, 1987). Los individuos examinados se colectaron en cultivos de caña de azúcar, arroz, maíz y en Prosopis pallida (Humb. \& Bonpl. ex. Wild.) Kunth (Fabaceae).

Superfamilia Cynipoidea Latreille, 1802+

Familia Figitidae Thomson, 1862+

Subfamilia Eucoilinae Westwood, 1835+

Género Ganaspidium Weld, $1952+$

3. Ganaspidium $\mathrm{sp}+$

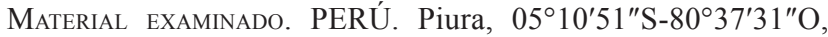
$29 \mathrm{msnm}$, Bosque estacionalmente seco de llanura.

Comentario. El género Ganaspidium es de distribución neotropical, sus especies son endoparasitoides de huevos en especies de Agromyzidae (Diptera) que se caracterizan por ser plagas de diversos de cultivos (Buffington, 2010). En Perú las especies de Ganaspidium son endoparasitoides de especies de plaga de Liriomyza Mik, 1894 y Japanagromyza Sasakawa, 1958 en cultivos de alfalfa, arveja, haba, zapallo, pepino, entre otros (Mujica \& Kroschel, 2011).

Referencia. Mujica \& Kroschel (2011).

Superfamilia Chalcidoidea Latreille, 1817

Familia Aphelinidae Thomson, 1876

Subfamilia Coccophaginae Förster, 1878

Género Encarsia Förster, 1878

4. Encarsia tabacivora Viggiani, 1895

Material examinado. PERÚ. 1 đo, Piura, La Arena, $05^{\circ} 20^{\prime} 34^{\prime \prime} \mathrm{S}-80^{\circ} 42^{\prime} 30^{\prime \prime} \mathrm{O}, \quad 29 \mathrm{msnm}, \quad 01-\mathrm{IV}-2017$, red entomológica, G.Juárez, Bosqueestacionalmentesecodellanura,

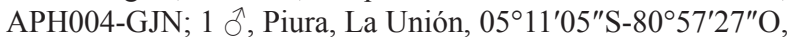
29 msnm, 01-I-2018, red entomológica, G. Juárez, Bosque estacionalmente seco de llanura, APH005-GJN; 2 ổ $^{\lambda}$, Piura, Castilla, $05^{\circ} 11^{\prime} 05^{\prime \prime} \mathrm{S}-80^{\circ} 57^{\prime} 27^{\prime \prime} \mathrm{O}, 29 \mathrm{msnm}$, 01-IV-2016, red entomológica, U. González, Bosque estacionalmente seco de llanura, APH006-GJN; Piura, 
Catacaos, $05^{\circ} 15^{\prime} 55^{\prime \prime} \mathrm{S}-80^{\circ} 40^{\prime} 30^{\prime \prime} \mathrm{O}, 29 \mathrm{msnm}, 01-\mathrm{IV}-2016$, red entomológica, G. Juárez, Bosque estacionalmente seco de llanura, APH007-GJN.

Comentario. Mencionada como Encarsia sp en Juárez-Noé \& González-Coronado (2018). Especie que se distribuye en América, Asia y Oceanía, es parasitoide de huevos y ninfas de especies de Aleyrodidae (Hemiptera) (Myartseva \& Evans, 2007). En Perú se utiliza para el control biológico de especies plaga como Bemisia tabaci (Gennadius, 1889) y A. floccosus conocidas como "moscas blancas" en diversos cultivos (Beingolea, 1959; Supanta-Huarcaya, 2017). Los individuos examinados se colectaron en cultivos de yuca, camote y frijol.

5. Encarsia formosa Gahan, $1924+$

Material examinado. PERÚ. 1 o, Piura, Castilla, Universidad Nacional de Piura, $05^{\circ} 10^{\prime} 51^{\prime \prime} \mathrm{S}-80^{\circ} 37^{\prime} 31^{\prime \prime} \mathrm{O}, 29 \mathrm{msnm}, 2-\mathrm{II}-$ 2020, red entomológica, G. Juárez, Bosque estacionalmente seco de llanura; 1 \% , Sullana, Cabo Verde, $04^{\circ} 51^{\prime} 27^{\prime \prime} \mathrm{S}-80^{\circ} 40^{\prime} 52^{\prime \prime} \mathrm{O}$, 23 msnm, 16-V-2020, red entomológica, G. Juárez, Bosque estacionalmente seco de llanura; 1 , , Sullana, Miguel Checa, $04^{\circ} 54^{\prime} \mathrm{S}-80^{\circ} 48^{\prime} \mathrm{O}, 23 \mathrm{msnm}, 24-\mathrm{XI}-2019$, red entomológica, G. Juárez, Bosque estacionalmente seco de llanura.

COMENTARIO. Especie cosmopolita que parasita huevos y ninfas de moscas blancas, especialmente de B. tabaci, A. floccosus, entre otros (Myartseva \& Evans, 2007; Supanta-Huarcaya, 2017). Los individuos examinados se colectaron en cultivos de yuca.

6. Encarsia pergandiella Howard, $1907+$

Material examinado. PERÚ. 1 , Piura, Castilla, Universidad NacionaldePiura, $05^{\circ} 10^{\prime} 51^{\prime \prime} \mathrm{S}-80^{\circ} 37^{\prime} 31^{\prime \prime} \mathrm{O}, 29 \mathrm{msnm}, 2-\mathrm{II}-2020$, red entomológica, U. González, Bosque estacionalmente seco de llanura; 1 ㅇ, Sullana, Cabo Verde, 04 $51^{\circ} 27^{\prime \prime} \mathrm{S}-80^{\circ} 40^{\prime} 52^{\prime \prime} \mathrm{O}$, 23 msnm, 16-V-2020, red entomológica, G. Juárez, Bosque estacionalmente seco de llanura; 1 ․ Sullana, Miguel Checa, $04^{\circ} 54^{\prime} \mathrm{S}-80^{\circ} 48^{\prime} \mathrm{O}, 23 \mathrm{msnm}, 24-\mathrm{XI}-2019$, red entomológica, G. Juárez, Bosque estacionalmente seco de llanura.

Comentario. Especie que se distribuye en Oceanía, América, Europa y Asia (Myartseva \& Evans, 2007). Es parasitoide de huevos y ninfas de moscas blancas, especialmente de $B$. tabaci, A. floccosus y otros (Myartseva \& Evans, 2007; SupantaHuarcaya, 2017). Los individuos examinados se colectaron en cultivo de yuca.

Subfamilia Aphelininae Thomson

Género Aphytis Howard, 1900

7. Aphytis chrysomphali Mercet, 1912

Material eXAMINAdo. PERÚ. 1 đ̄, Paita, Vichayal, 04 51'55"S-81 04'19"O, $10 \mathrm{msnm}, \quad 11-\mathrm{X}-2017$, trampa malaise, G. Juárez, Bosque estacionalmente seco de llanura; 1 ô, Paita, $04^{\circ} 04^{\prime} \mathrm{S}-81^{\circ} 06^{\prime} \mathrm{O}, 10 \mathrm{msnm}, 11-\mathrm{X}-2017$, trampa malaise, G. Juárez, Bosque estacionalmente seco de llanura.

Comentario. Especie cosmopolita, ectoparasitoide en especies de Diaspididae (Hemiptera) plagas en diversos cultivos y frutales (Myartseva et al., 2010). En Perú se reporta parasitando Selenaspidus articulatus (Morgan, 1889) "queresa redonda de los cítricos" (Beingolea, 1967; 1969). Especie que amplía su área de distribución conocida dentro de la región a la provincia de Paita, anteriormente citada para las provincias de Piura y Sullana (Juárez-Noé \& González-Coronado, 2018). Los individuos examinados se colectaron en cultivos de limón.

Subfamilia Eretmocerinae Shafee \& Khan, $1978+$

Género Eretmocerus Haldeman, $1850+$

8. Eretmocerus eremicus Rose \& Zolnerowich, $1997+$

Material eXaminado. PERÚ. 2 우, Piura, Castilla, Universidad Nacional de Piura, $05^{\circ} 10^{\prime} 51^{\prime \prime} \mathrm{S}-80^{\circ} 37^{\prime} 31^{\prime \prime} \mathrm{O}, 29 \mathrm{msnm}, 2$-III-
2020, red entomológica, G. Juárez, Bosque estacionalmente seco de llanura.

Comentario. Especie parasitoide en especies de Aleyrodidae (Hemiptera), en Perú se reporta de Trialeurodes variabilis (Quaintance, 1990) plaga en M. sculenta (Aliaga, 2012). Los individuos examinados se colectaron en cultivo de yuca.

Familia Chalcididae Latreille, 1817

Subfamilia Chalcidinae Latreille, 1817

Género Brachymeria Westwood, 1829

9. Brachymeria podagrica (Fabricius, 1787)

Material eXaminado. PERÚ. 1 q, Piura, La Arena, 05'20'34"S-80 42'30"O, 29 msnm, 17-I-2017, red entomológica, U. González, Bosque estacionalmente seco de llanura, CHAL002-GJN; 1 , Sullana, Lancones,

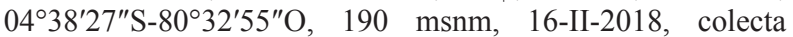
manual, G. Juárez, Bosque estacionalmente seco de colina, CHAL003-GJN; 2 oq , Talara, Los Órganos, 04 $10^{\prime} 38^{\prime \prime} \mathrm{S}-81^{\circ} 07^{\prime} 27^{\prime \prime} \mathrm{O}, 32 \mathrm{msnm}, 19-\mathrm{VIII}-2017$, red entomológica, G. Juárez, Bosque estacionalmente seco de llanura, CHAL003-GJN; $1 \partial^{\lambda}$, Paita, $05^{\circ} 04^{\prime} \mathrm{S}-81^{\circ} 06^{\prime} \mathrm{O}$, 10 msnm, 09-IV-2017, trampa red entomológica, U. González, Bosque estacionalmente seco de llanura, CHAL004-GJN; $1 \hat{\jmath}$, Sechura, Sechura, $05^{\circ} 34^{\prime} \mathrm{S}-81^{\circ} 16^{\prime} \mathrm{O}, 16 \mathrm{msnm}, 22-\mathrm{V}-$ 2016, red entomológica, G. Juárez, Bosque estacionalmente seco de llanura, CHAL005-GJN; 1 9, Morropón, Chulucanas, $05^{\circ} 05^{\prime} \mathrm{S}-80^{\circ} 99^{\prime} \mathrm{O}, 350 \mathrm{msnm}, 10-\mathrm{VI}-2016$, red entomológica, U. González, Bosque estacionalmente seco de colina, CHAL006-

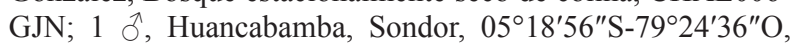
1.755 msnm, 12-IX-2018, colecta manual, G. Juárez, Bosque seco interandino, CHAL007-GJN; 1 $\hat{\text {, Ayabaca, }}$

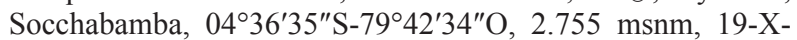
2017, colecta manual, G. Juárez, Bosque húmedo de montaña, CHAL008-GJN.

Comentario. Mencionada como Brachymeria sp en Juárez-Noé \& González-Coronado (2018). Especie cosmopolita que en la región neotropical se distribuye en México, Haití, Jamaica, Brasil, Argentina y Perú. Es endoparasitoide en larvas de especies de Muscidae, Sarcophagidae y Calliphoridae (Delvare \& Huchet, 2017). Los individuos examinados se colectaron en cuerpo en descomposición de cerdo y en árboles $P$. pallida.

Familia Encyrtidae Walker, $1837+$

Género Ooencyrtus Ashmead, $1990+$

10. Ooencyrtus bucculatrix (Howard, 1883) +

Material examinado. PERÚ. Piura, $05^{\circ} 10^{\prime} 51^{\prime \prime S} \mathrm{~S}-80^{\circ} 37^{\prime} 31^{\prime \prime} \mathrm{O}$, $29 \mathrm{msnm}$, Bosque estacionalmente seco de llanura.

Comentario. Especie de distribución americana, en Perú es parasitoide de larvas de Bucculatrix thurberiella Busck, 1914 (Bucculatricidae) "gusano perforador de la hoja" en cultivos de algodón en la costa norte del país (Herrera \& Álvarez, 1979).

REFERENCIA. Herrera \& Álvarez (1979).

Familia Eulophidae Westwood, 1829

Subfamilia Entedoninae Förster, 1856

Género Chrysocharis Förster, 1856

11. Chrysocharis vonones (Walker, 1839)

Material examinado. Perú. 1 +1 0 , Piura, Castilla, Universidad Nacional de Piura, $05^{\circ} 10^{\prime} 51^{\prime \prime} \mathrm{S}-80^{\circ} 37^{\prime} 31^{\prime \prime} \mathrm{O}$, 29 msnm, 12-II-2017, red entomológica, G. Juárez, Bosque estacionalmente seco de llanura, EUL001-GJN; $1 \delta^{\lambda}$, Piura, Catacaos, $05^{\circ} 15^{\prime} 42^{\prime \prime} \mathrm{S}-80^{\circ} 40^{\prime} 27^{\prime \prime} \mathrm{O}, 29 \mathrm{msnm}$, 15-II-2018, red entomológica, U. González, Bosque estacionalmente seco de llanura, EUL002-GJN; 1 ㅇ, Sullana, Salitral, $04^{\circ} 51^{\prime} 27^{\prime \prime} \mathrm{S}-80^{\circ} 40^{\prime} 52^{\prime \prime} \mathrm{O}, 23 \mathrm{msnm}, 06-\mathrm{III}-$ 2016, red entomológica, G. Juárez, Bosque estacionalmente 
seco de llanura, EUL003-GJN; 1 , Talara, Lobitos, $04^{\circ} 27^{\prime} 10^{\prime \prime} \mathrm{S}-81^{\circ} 16^{\prime} 40^{\prime \prime} \mathrm{O}, \quad 12 \mathrm{msnm}, 27-\mathrm{IV}-2017$, red entomológica, G. Juárez, Bosque estacionalmente seco de llanura, EUL004-GJN; 1 ㅇ, El Alto, $04^{\circ} 16^{\prime} 04^{\prime \prime} \mathrm{S}-81^{\circ} 13^{\prime} 09^{\prime \prime} \mathrm{O}$, 300 msnm, 01-IV-2017, red entomológica, G. Juárez, Bosque estacionalmente seco de colina, EUL005-GJN; 1 q, Paita, Paita, $05^{\circ} 04^{\prime} \mathrm{S}-81^{\circ} 06^{\prime} \mathrm{O}, 10 \mathrm{msnm}, 27-\mathrm{V}-2017$, trampa Malaise, U. González, Bosque estacionalmente seco de llanura,

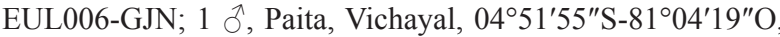
10 msnm, 21-IV-2018, trampa de intercepción, G. Juárez, Bosque estacionalmente seco de llanura, EUL007-GJN; $2 \delta^{\lambda}$, Sechura, Desierto de Sechura, $05^{\circ} 34^{\prime} \mathrm{S}-81^{\circ} 16^{\prime} \mathrm{O}, 10$ msnm, 17-X-2018, trampa de intercepción, G. Juárez, Bosque estacionalmente seco de llanura, EUL008-GJN.

Comentario. Mencionada como Chrysocharis sp en Juárez-Noé \& González-Coronado (2018). Especie parasitoide en especies de Agromyzidae especialmente del género Liriomyza que se comportan como plagas en diversos cultivos en el mundo (Mujica \& Kroschel, 2011; Cardoso da Costa-Lima et al., 2014). Algunos de los individuos examinados se colectaron en cultivos de yuca y camote árboles de $P$. pallida.

Género Proacrias Ihering, $1914+$

12. Proacrias thysanoides (De Santis, 1972) +

Material examinado. PERÚ. Piura, 0510'51"S-80³7'31"O, 29 msnm, Bosque estacionalmente seco de llanura.

Comentario. Especie de distribución neotropical parasitoide de huevos y larvas de especies de Lepidoptera y Diptera. En Perú se reporta como parasitoide de especies de Agromyzidae especialmente de los géneros Liriomyza y Japanagromyza (Reina \& La Salle, 2003; Mujica \& Kroschel, 2011).

REFERENCIA. Mujica \& Kroschel (2011).

Subfamilia Eulophinae Westwood, 1829

Género Zagrammosoma Ashmead, $1904+$

13. Zagrammosoma variegatum (Masi, 1907) +

Material examinado. PERÚ. Piura, 05¹0'51"S-80³7'31"O, 29 msnm, Bosque estacionalmente seco de llanura.

Comentario. Especie de amplia distribución mundial, ectoparasitoide de huevos y larvas de especies minadores de hojas de coleópteros, lepidópteros y dípteros. En Perú se reporta como parasitoide de especies de Agromyzidae especialmente de los géneros Liriomyza y Japanagromyza (Reina \& La Salle, 2003; Mujica \& Kroschel, 2011).

REFERENCIA. Herrera \& Álvarez (1979).

Género Diglyphus Walker, $1844+$

14. Diglyphus websteri (Crawford, 1912) +

Material examinado. PERÚ. Piura, 0510'51"S-80³7'31"O, $29 \mathrm{msnm}$, Bosque estacionalmente seco de llanura.

Comentario. Especie parasitoide de especies de Agromyzidae especialmente de los géneros Liriomyza y Japanagromyza (Mujica \& Kroschel, 2011).

REFERENCIA. Mujica \& Kroschel (2011).

Género Diaulinopsis Crawford, $1912+$

15. Diaulinopsis callichroma Crawford, $1912+$

Material examinado. Perú. Piura, 0510'51"S-80³7'31"O, $29 \mathrm{msnm}$, Bosque estacionalmente seco de llanura.

Comentario. Especie parasitoide de especies de Agromyzidae especialmente de los géneros Liriomyza y Japanagromyza (Mujica \& Kroschel, 2011).

ReFerencia. Mujica \& Kroschel (2011).

Familia Pteromalidae Dalman, 1820

Subfamilia Pteromalinae Dalman, 1820
Género Dibrachys Förster, 1856

16. Dibrachys microgastri (Bouché, 1834)

Material examinado. PERÚ. 1 o, Piura, Castilla, Universidad Nacional de Piura, $05^{\circ} 10^{\prime} 51^{\prime \prime} \mathrm{S}-80^{\circ} 37^{\prime} 31^{\prime \prime} \mathrm{O}, 29 \mathrm{msnm}, 12-\mathrm{II}-$ 2018, red entomológica, G. Juárez, Bosque estacionalmente seco de llanura, PTER001-GJN; 1 \%, Piura, Catacaos, $05^{\circ} 15^{\prime} 42^{\prime \prime} \mathrm{S}-80^{\circ} 40^{\prime} 27^{\prime \prime} \mathrm{O}, 29 \mathrm{msnm}, \quad 15-\mathrm{IV}-2018$, red entomológica, U. González, Bosque estacionalmente seco de llanura, PTER002-GJN; 1 †, Sullana, Salitral, 04 51'27"S-8040'52"O, 23 msnm, 06-III-2017, red entomológica, G. Juárez, Bosque estacionalmente seco de llanura, PTER004-GJN.

Comentario. Mencionada como Dibrachys sp en Juárez-Noé \& González-Coronado (2018). Especie de distribución mundial, ectoparasitoide larval en especies de Diptera, Hymenoptera, Lepidoptera y Coleoptera (Peters \& Baur, 2011). Los individuos examinados se colectaron en P. pallida.

Subfamilia Miscogastrinae Walker, 1833+

Género Halticoptera Spinola, 1811+

17. Halticoptera arduine (Walker, 1843) +

Material examinado. Perú. Piura, 05¹0'51"S-80³7'31"O, $29 \mathrm{msnm}$, Bosque estacionalmente seco de llanura.

Comentario. Especie reportada en Costa Rica, Argentina, Chile, Bolivia, Ecuador y Perú e introducida en Kenia (Kroschel et al., 2016). En Perú se reporta como endoparasitoide de larvas en especies de Agromyzidae especialmente de los géneros Liriomyza, Japanagromyza y Melanagromyza Hendel, 1920 (Mujica \& Kroschel, 2011; Kroschel et al., 2016).

ReFERENCIA. Mujica \& Kroschel (2011).

Familia Torymidae Walker, $1833+$

Género Podagrion Spinola, $1811+$

18. Podagrion brasiliense Howard, $1894+$

Material examinado. PERÚ. 1 , Piura, Castilla, Universidad Nacional de Piura, $05^{\circ} 10^{\prime} 51^{\prime \prime} \mathrm{S}-80^{\circ} 37^{\prime} 31^{\prime \prime} \mathrm{O}, 29 \mathrm{msnm}, 12-\mathrm{II}-$ 2018, trampa malaise, G. Juárez, Bosque estacionalmente seco de llanura, PTER001-GJN

Comentario. Especie de distribución neotropical que en Perú ha sido registrado como endoparasitoide en ootecas del género Musonia Stål, 1887 (Mantodea) (Rivera, 2003).

Género Megastigmus Dalman, $1820+$

19. Megastigmus transvaalensis (Hussey, 1956) + (Fig. 1)

Material examinado. PERÚ. 1, 1 $\widehat{\delta}$, Piura, Castilla, Universidad Nacional de Piura, $05^{\circ} 10^{\prime} 51^{\prime \prime} \mathrm{S}-80^{\circ} 37^{\prime} 31^{\prime \prime} \mathrm{O}, 29 \mathrm{msnm}, 12-\mathrm{II}-$ 2019, red entomológica, G. Juárez, Bosque estacionalmente seco de llanura; $1 \delta^{\lambda}$, Piura, Catacaos, $05^{\circ} 15^{\prime} 42^{\prime \prime} \mathrm{S}-80^{\circ} 40^{\prime} 27^{\prime \prime} \mathrm{O}$, 29 msnm, 15-II-2020, red entomológica, U. González, Bosque estacionalmente seco de llanura; 1 , Sullana, Salitral, 04 $51^{\prime} 27^{\prime \prime} \mathrm{S}-80^{\circ} 40^{\prime} 52^{\prime \prime} \mathrm{O}, 23 \mathrm{msnm}, 06-\mathrm{III}-2019$, red entomológica, G. Juárez, Bosque estacionalmente seco de llanura; 1 ô, 1 o, Talara, Lobitos, $04^{\circ} 27^{\prime} 10^{\prime \prime} \mathrm{S}-81^{\circ} 16^{\prime} 40^{\prime \prime} \mathrm{O}$, 12 msnm, 27-IV-2017, red entomológica, G. Juárez, Bosque estacionalmente seco de llanura; 1 क, El Alto, 04 $16^{\prime} 04^{\prime \prime} \mathrm{S}-81^{\circ} 13^{\prime} 09^{\prime \prime} \mathrm{O}, \quad 300 \mathrm{msnm}, \quad 01-\mathrm{V}-2019$, red entomológica, G. Juárez, Bosque estacionalmente seco de colina; 1 ㅇ, Paita, Paita, $05^{\circ} 04^{\prime} \mathrm{S}-81^{\circ} 06^{\prime} \mathrm{O}, 10 \mathrm{msnm}, 27-\mathrm{V}-$ 2017, red entomológica, U. González, Bosque estacionalmente seco de llanura; $1{ }^{\lambda}$, Paita, Vichayal, $04^{\circ} 51^{\prime} 55^{\prime \prime} \mathrm{S}-81^{\circ} 04^{\prime} 19^{\prime \prime} \mathrm{O}$, 10 msnm, 21-VII-2019, red entomológica, G. Juárez, Bosque estacionalmente seco de llanura; $2 \hat{\jmath} \hat{\jmath}$, Sechura, Sechura, $05^{\circ} 34^{\prime} \mathrm{S}-81^{\circ} 16^{\prime} \mathrm{O}, 10 \mathrm{msnm}, 17-\mathrm{XI}-2019$, red entomológica, G. Juárez, Bosque estacionalmente seco de llanura.

Comentario. Especie de distribución mundial caracterizada por atacar frutos y semillas de especies de Anacardiaceae y 
Pinaceae (Roques \& Skrzypcznska, 2003; Fernandes et al., 2014). En el neotrópico ha sido registrada atacando frutos y semillas de molle (Fernandes et al., 2014), Los individuos examinados se colectaron sobre hojas molle.

Familia Trichogrammatidae Haliday, 1851

Género Trichogramma Westwood

20. Trichogramma (Trichogramma) pretiosum Riley, 1879

Material examinado. 1 o, Morropón, Piedra del Toro, $05^{\circ} 11^{\prime} \mathrm{S}-80^{\circ} 49^{\prime} \mathrm{O}, 1.225 \mathrm{msnm}, 22-\mathrm{XI}-2019$, trampa Malaise, G. Juárez, Bosque estacionalmente seco de colina; 1 ㅇ, Morropón, $05^{\circ} 05^{\prime} \mathrm{S}-80^{\circ} 99^{\prime} \mathrm{O}, 1.225 \mathrm{msnm}, 19-\mathrm{XII}-2019$, trampa Malaise, G. Juárez, Bosque estacionalmente seco de colina; 1 9, Morropón, Buenos Aires, $05^{\circ} 05^{\prime} 51^{\prime \prime} \mathrm{S}-80^{\circ} 09^{\prime} 44^{\prime \prime} \mathrm{O}$, 1.225 msnm, 13-IX-2019, trampa Malaise, G. Juárez, Bosque estacionalmente seco de colina; 1 , Morropón, Santa Catalina de Mossa, 0506'10"S79 53'06"O, 1.225 msnm, 11-V-2019, trampa Malaise, G. Juárez, Bosque húmedo de montaña.

Comentario. Especie que se distribuye desde el sudeste de Canadá hasta Argentina, con introducciones en Hawai (USA) y Australia (Velásquez de Ríos \& Terán, 2003). Es parasitoide de especies de lepidópteros de los géneros Erinnyis Hübner, 1807, Helicoverpa, Alabama Grote, 1895 y Anomis Hübner, 1821 que actúan como placas en cultivos de yuca, maíz y algodón (Whu \& Valdivieso, 1999; Velásquez de Ríos \& Terán, 2003). Especie que amplía su área de distribución conocida dentro de la región a la provincia de Morropón, anteriormente registrada en las provincias de Piura, Sullana, Paita, Talara, Sechura, Huancabamba, Ayabaca (Juárez-Noé \& González-Coronado, 2018). Los individuos examinados se colectaron en cultivo maíz.

21. Trichogramma (Trichogramma) exiguum Pinto \& Platner, 1978

Material examinado. Perú. 1 क, Morropón, Piedra del Toro, $05^{\circ} 11^{\prime} \mathrm{S}-80^{\circ} 49^{\prime} \mathrm{O}, 1.225 \mathrm{msnm}, 22-\mathrm{XI}-2019$, trampa Malaise, G. Juárez, Bosque estacionalmente seco de colina; 1 ㅇ, Morropón, $05^{\circ} 05^{\prime} \mathrm{S}-80^{\circ} 99^{\prime} \mathrm{O}, 1.225 \mathrm{msnm}, 19-\mathrm{XII}-2019$, trampa Malaise, G. Juárez, Bosque estacionalmente seco de colina; 1 q, Morropón, Buenos Aires, $05^{\circ} 05^{\prime} 51^{\prime \prime} \mathrm{S}-80^{\circ} 09^{\prime} 44^{\prime \prime} \mathrm{O}$, 1.225 msnm, 13-IX-2019, trampa Malaise, G. Juárez, Bosque estacionalmente seco de colina; 1 ㅇ, Morropón, Santa Catalina de Mossa, 0506'10"S79 53'06"O, 1.225 msnm, 11-V-2019, trampa Malaise, G. Juárez, Bosque húmedo de montaña.

Comentario. Especie que se distribuye en Norte, Centro y Sur América (Velásquez de Ríos \& Terán, 2003). Es parasitoide de especies de lepidópteros de los géneros Helicoverpa Hardwick, 1965, Spodoptera Guenée, 1852, Heliothis Ochsenheimer, 1816, Diatraea Guilding, 1828 y Manduca Hübner, 1807 que actúan como plagas en cultivos de maíz, caña de azúcar, tomate y algodón (Whu \& Valdivieso, 1999; Velásquez de Ríos \& Terán, 2003). Especie que amplía su área de distribución conocida dentro de la región a la provincia de Morropón, anteriormente registrada en las provincias de Piura, Sullana, Paita, Talara, Sechura, Huancabamba, Ayabaca (Juárez-Noé \& González-Coronado, 2018). Los individuos examinados se colectaron en cultivo maíz.

Superfamilia Ichneumonoidea Latreille, 1802

Familia Braconidae Nees, 1811

Subfamilia Microgastrinae Förster, 1862

Género Cotesia Cameron, 1891

22. Cotesia flavipes Cameron,

Material eXAminado. PERÚ. 1 ते, Piura, Catacaos, 05 $15^{\prime} 42^{\prime \prime} \mathrm{S}-80^{\circ} 40^{\prime} 27^{\prime \prime} \mathrm{O}, \quad 29 \mathrm{msnm}, \quad 25-\mathrm{IV}-2017$, red entomológica, U. González, Bosque estacionalmente seco de llanura, BRAC022-GJN; 1 \&, Sullana, Salitral, 0451'27"S-80 40'52"O, $23 \mathrm{msnm}, 16-\mathrm{V}-2017$, red entomológica, G. Juárez, Bosque estacionalmente seco de llanura, BRA032.

Comentario. Mencionada como Cotesia sp en Juárez-Noé \& González-Coronado (2018). Especie de origen Indo Oriental e introducido a países a nivel mundial por ser endoparasitoide de larvas en especies de lepidópteros que actúan como plagas en diversos cultivos (Osorio-Mejía, 2018). En América se le usa en el control biológico de $D$. saccharalis plaga en caña de azúcar (Redolfi de Huiza, 1994; Osorio-Mejía, 2018; AstolaMariscal \& Narrea-Cango, 2019). Los individuos examinados se colectaron en cultivo de caña de azúcar.

Género Glyptapanteles Ashmead, 1904

23. Glyptapanteles muesebecki (Blanchard, 1947)

Material examinado. PERÚ. 1 +, 1 , Ayabaca, Suyo, $04^{\circ} 30^{\prime} 45^{\prime \prime} \mathrm{S}-80^{\circ} 02^{\prime} 10^{\prime \prime} \mathrm{O}, 450 \mathrm{msnm}, \quad 26-\mathrm{I}-2020$, trampa de intercepción, trampa malaise, G. Juárez, Bosque estacionalmente seco de colina.

Comentario. Especie que se distribuye en Argentina y Perú. Es parasitoide de especies del género Mythimna Ochsenheimer, 1816 (Noctuidae) plagas en cultivos de arroz y maíz principalmente (Redolfi de Huiza, 1994). Especie que amplía su área de distribución conocida dentro de la región a la provincia de Ayabaca, anteriormente registrada en las provincias de Piura, Sullana, Talara, Paita, Sechura, Morropón (Juárez-Noé \& González-Coronado, 2018). Los individuos examinados se colectaron en cultivo de maíz.

Subfamilia Cheloninae Förster, 1862

Género Microchelonus Szpligeti, 1908

24. Microchelonus townsendi (Viereck, 1912) *

Material examinado. PERÚ. 2 9 , 1 $\hat{\jmath}$, Ayabaca, Suyo, $04^{\circ} 30^{\prime} 45^{\prime \prime} \mathrm{S}-80^{\circ} 02^{\prime} 10^{\prime \prime} \mathrm{O}, 450 \mathrm{msnm}, 03-\mathrm{II}-2020$, trampa de intercepción, trampa Malaise, G. Juárez, Bosque estacionalmente seco de colina.

Comentario. Especie parasitoide de Anthonomus vestitus Boheman, 1859 (Curculionidae) plaga en cultivo de algodón (Redolfi de Huiza, 1994). Especie que amplía su área de distribución conocida dentro de la región a la provincia de Ayabaca, anteriormente registrada para las provincias de Piura, Sullana, Paita, Sechura, Talara, Morropón (Juárez-Noé \& González-Coronado, 2018). Los individuos examinados se colectaron en cultivo de maíz y algodón.

Subfamilia Helconinae Förster, 1862

Género Triaspis Haliday, 1835

25. Triaspis vestiticida Viereck, 1912

Material eXAminado. PERÚ. 1 đo, Talara, Pariñas, 043' $52^{\prime \prime} \mathrm{S}-81^{\circ} 16^{\prime} 29^{\prime \prime} \mathrm{O}, 12 \mathrm{msnm}, 17-\mathrm{V}-2019$, trampa de intercepción, G. Juárez, Bosque estacionalmente seco de llanura; 1 ㅇ, Máncora, $04^{\circ} 06^{\prime} 26^{\prime \prime} \mathrm{S}-81^{\circ} 02^{\prime} 50^{\prime \prime} \mathrm{O}$, 300 msnm, 11-VI-2019, trampa de intercepción, G. Juárez, Bosque estacionalmente seco de colina; $1 \hat{\partial}$, Sechura, Vice, $05^{\circ} 25^{\prime} \mathrm{S}-81^{\circ} 46^{\prime} \mathrm{O}, 10 \mathrm{msnm}, 27-\mathrm{VIII}-2019$, trampa de intercepción, G. Juárez, Bosque estacionalmente seco de llanura; 1 ふै, Sechura, Cristo nos Valga, $05^{\circ} 29^{\prime} 37^{\prime \prime} \mathrm{S}-80^{\circ} 44^{\prime} 28^{\prime \prime} \mathrm{O}$, 10 msnm, 27-VIII-2019, trampa de intercepción, G. Juárez, Bosque estacionalmente seco de llanura 1 q, Morropón,

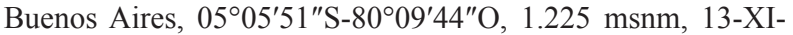
2019, trampa Malaise, U. González, Bosque estacionalmente seco de colina.

Comentario. Especie parasitoide de $A$. vestitus plaga en cultivo de algodón (Redolfi de Huiza, 1994). Especie que amplía su área de distribución conocida dentro de la región a las provincias de 
Talara, Morropón y Sechura, anteriormente registrada para las provincias de Piura, Sullana, Paita (Juárez-Noé \& GonzálezCoronado, 2018). Los individuos examinados se colectaron en cultivo algodón.

Subfamilia Rogadinae Förster, 1862

Género Aleiodes Wesmael, 1838

26. Aleiodes gossypii (Muesebeck, 1960)

Material EXAMINAdO. PERÚ. 1 ô, Talara, Pariñas, 0434'52"S-81 $16^{\circ} 29^{\prime \prime} \mathrm{O}, 12 \mathrm{msnm}, 17-\mathrm{V}-2019$, trampa de intercepción, G. Juárez, Bosque estacionalmente seco de llanura; 1 ․ Máncora, $04^{\circ} 06^{\prime} 26^{\prime \prime} \mathrm{S}-81^{\circ} 02^{\prime} 50^{\prime \prime} \mathrm{O}, 300 \mathrm{msnm}, 11-\mathrm{VI}-2019$, trampa de intercepción, G. Juárez, Bosque estacionalmente seco de colina; 1 ㅇ, Paita, Paita, $05^{\circ} 04^{\prime} \mathrm{S}-81^{\circ} 06^{\prime} \mathrm{O}, 10 \mathrm{msnm}, 12$-VII2019, trampa Malaise, U. González, Bosque estacionalmente

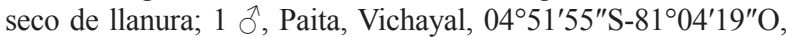
10 msnm, 20-XII-2019, trampa Malaise, G. Juárez, Bosque estacionalmente seco de llanura.

Comentario. Especie que se distribuye en Brasil y Perú. Es parasitoide de Anomis texana Riley, 1885 (Erebidae) y Alabama argillacea (Hübner, 1823) (Erebidae) plagas en cultivo de algodón (Redolfi de Huiza, 1994). Especie que amplía su área de distribución conocida dentro de la región a las provincias de Paita y Talara, anteriormente registrada para las provincias de Piura, Sullana (Juárez-Noé \& González-Coronado, 2018). Los individuos examinados se colectaron en cultivo de algodón.

Familia Ichneumonidae Latreille, 1802

Subfamilia Ophioninae Shuckard, 1840

Género Enicospilus Stephens, 1835

27. Enicospilus purgatus (Say, 1835)

Material examinado. PERÚ. 1 \&, Piura, La Arena, 05²0'34"S-80 42'30"O, 29 msnm, 27-IV-2018, red entomológica, G. Juárez, Bosque estacionalmente seco de llanura, ICH010-GJN; 1 q, Sullana, Lancones, $04^{\circ} 38^{\prime} 27^{\prime \prime} \mathrm{S}-80^{\circ} 32^{\prime} 55^{\prime \prime} \mathrm{O}$, 190 msnm, 12-V-2018, red entomológica, G. Juárez, Bosque estacionalmente seco de colina, ICH012-GJN; 1 ㅇ, Talara, Los Órganos, $04^{\circ} 10^{\prime} 38^{\prime \prime} \mathrm{S}-81^{\circ} 07^{\prime} 27^{\prime \prime} \mathrm{O}, 32 \mathrm{msnm}, 22-\mathrm{VII}-$ 2017, red entomológica, G. Juárez, Bosque estacionalmente seco de llanura, ICH014-GJN; $1 \partial^{\lambda}$, Paita, $05^{\circ} 04^{\prime} \mathrm{S}-81^{\circ} 06^{\prime} \mathrm{O}$, 10 msnm, 13-VII-2018, red entomológica, U. González, Bosque estacionalmente seco de llanura, ICH017-GJN; 1 oे, Sechura, Sechura, $05^{\circ} 34^{\prime} \mathrm{S}-81^{\circ} 16^{\prime} \mathrm{O}, 16 \mathrm{msnm}, 26-\mathrm{IV}-2016$, red entomológica, G. Juárez, Bosque estacionalmente seco de llanura, ICH022-GJN; 1 + , Paita, Paita, $05^{\circ} 04^{\prime} \mathrm{S}-81^{\circ} 06^{\prime} \mathrm{O}$, 10 msnm, 12-VII-2019, red entomológica, U. González, Bosque estacionalmente seco de llanura, ICH025-GJN.

Comentario. Mencionada como Enicospilus sp en Juárez-Noé \& González-Coronado (2018). Especie de distribución neártica y neotropical, es parasitoide principalmente de lepidópteros plaga en diversos cultivos (Rodríguez-Berrío, 2006). Los individuos examinados se colectaron en cultivos de maíz.

Género Brachycyrtus Kriechbaumer $1880+$

28. Brachycyrtus pretiosus Cushman, $1936+$

Material examinado. Perú. Piura, 05¹0'51"S-80³7'31"O, $29 \mathrm{msnm}$, Bosque estacionalmente seco de llanura.

Comentario. Especie que se distribuye desde el sur de Estados Unidos hasta Argentina (Onody et al., 2009). Es parasitoide de A. argillacea (Carrasco, 1972).

REFERENCIA. Carrasco (1972).

Género Isdromas Foerster, 1868+

29. Isdromas peruvianus (Viereck, 1912) +*

Material examinado. Perú. Piura, 05¹0'51"S-80³7'31"O, 29 msnm, Bosque estacionalmente seco de llanura.
COMENTARIO. Especie parasitoide de especies de lepidópteros plaga en diversos cultivos (Carrasco, 1972).

Referencia. Carrasco (1972).

Género Aeliopotes Porter, $1985+$

30. Aeliopotes paitensis (Cockerell, 1927) +*

Material examinado. PERÚ. Paita, Paita, $05^{\circ} 04^{\prime} \mathrm{S}-81^{\circ} 06^{\prime} \mathrm{O}$, $10 \mathrm{msnm}$, Bosque estacionalmente seco de llanura.

COMEnTARIo. Especie que se distribuye en la franja costera desértica desde la región Piura hasta la región Arequipa (Porter, 1985).

ReFERENCIA. Porter (1985).

Subfamilia Cryptinae Kirby, 1837

Género Cryptanura Brullé, 1846

31. Cryptanura sp (Fig. 2)

Material eXAminado. PERÚ. 1 , Huancabamba, Huancabamba, $05^{\circ} 27^{\prime} 07^{\prime \prime S}-79^{\circ} 36^{\prime} 22^{\prime \prime} \mathrm{O}, 2.567 \mathrm{msnm}, 15-\mathrm{VIII}-2019$, red entomológica, G. Juárez, Bosque húmedo de montaña; 1 +, Huancabamba, Canchaque, Cerro Huayanay, $05^{\circ} 24^{\prime} 07^{\prime \prime} \mathrm{S}-79^{\circ} 36^{\prime} 22^{\prime \prime} \mathrm{O}, \quad 1.600$ msnm, 16-VIII-2019, red entomológica, U. González, Bosque húmedo de montaña; $1 \hat{\sigma}$, Huancabamba, Canchaque, Pampa Minas, 05²1'20.6"S-79³5'20"O, 2.100 msnm, 03-XI-2019, red entomológica, U. González, Bosque húmedo de montaña; 1 సै, Huancabamba, Carmen de la Frontera, $05^{\circ} 08^{\prime} 54^{\prime \prime} \mathrm{S}-79^{\circ} 25^{\prime} 41^{\prime \prime} \mathrm{O}$, $2.200 \mathrm{msnm}, 17-\mathrm{XI}-2018$, red entomológica, U. González, Bosque húmedo de montaña; 1 ${ }^{\lambda}$, Huancabamba, Sondor, 05 $18^{\prime} 56^{\prime \prime} \mathrm{S}-79^{\circ} 24^{\prime} 36^{\prime \prime} \mathrm{O}, 2.100 \mathrm{msnm}, 02-\mathrm{X}-2019$, red entomológica, U. González, Bosque seco interandino; 1 गे, Ayabaca, Laguna el Tunal, $04^{\circ} 47^{\prime} 23^{\prime \prime} \mathrm{S}-79^{\circ} 50^{\prime} 42^{\prime \prime} \mathrm{O}$, 2.800 msnm, 04-XI-2019, red entomológica, G. Juárez, Bosque húmedo de montaña; 1 $\hat{\partial}$, Ayabaca, Yacupampa,

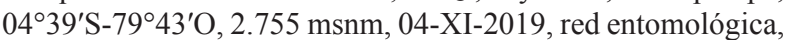
G. Juárez, Bosque húmedo de montaña; 1 , Ayabaca, Jililí, 04³8'13"S-7943'26"O, 450 msnm, 19-V-2019, red entomológica, G. Juárez, Bosque seco interandino.

COMENTARIo. El género Cryptanura es predominantemente neotropical teniendo la mayor diversidad de especies en Sur América (Kasparyan \& Ruíz-Cancino, 2006).

Superfamilia Chrysidoidea Latreille, 1802+

Familia Chrysididae Latreille, $1802+$

Subfamilia Chrysidinae Latreille, $1802+$

Género Holopyga Dalhbom, $1854+$

32. Holopyga $\mathrm{sp}+$

Material examinado. PERÚ. 1 ô, Piura, Castilla, Universidad Nacional de Piura, $05^{\circ} 10^{\prime} 51^{\prime \prime} \mathrm{S}-80^{\circ} 37^{\prime} 31^{\prime \prime} \mathrm{O}, 29 \mathrm{msnm}, 10$-II2020, red entomológica, U. González, Bosque estacionalmente seco de llanura.

Comentario. El género Holopyga se distribuye en todas las regiones zoogeográficas teniendo mayor diversidad de especies en la región Paleártica. Es parasitoide de especies de Sphecidae y Crabronidae (Farhad et al., 2017). El individuo examinado se colectó sobre hoja de Portulaca oleracea L. (Portulacaceae).

Superfamilia Apoidea Latreille, 1802

Familia Andrenidae Latreille, 1802

Subfamilia Panurginae Leach, 1815

Género Acamptopoeum Cockerell, $1905+$

33. Acamptopoeum (Acamptopoeum) vagans (Cockerell, 1926) +*

Material eXaminado. PERÚ. Piura, 05¹0'51"S-80³7'31"O, $29 \mathrm{msnm}$, Bosque estacionalmente seco de llanura.

Comentario. El género Acamptopoeum contiene ocho especies todas de distribución netamente neotropical (Colombia, Argentina, Paraguay, Uruguay, Chile y Perú) (Ruz, 1991). 

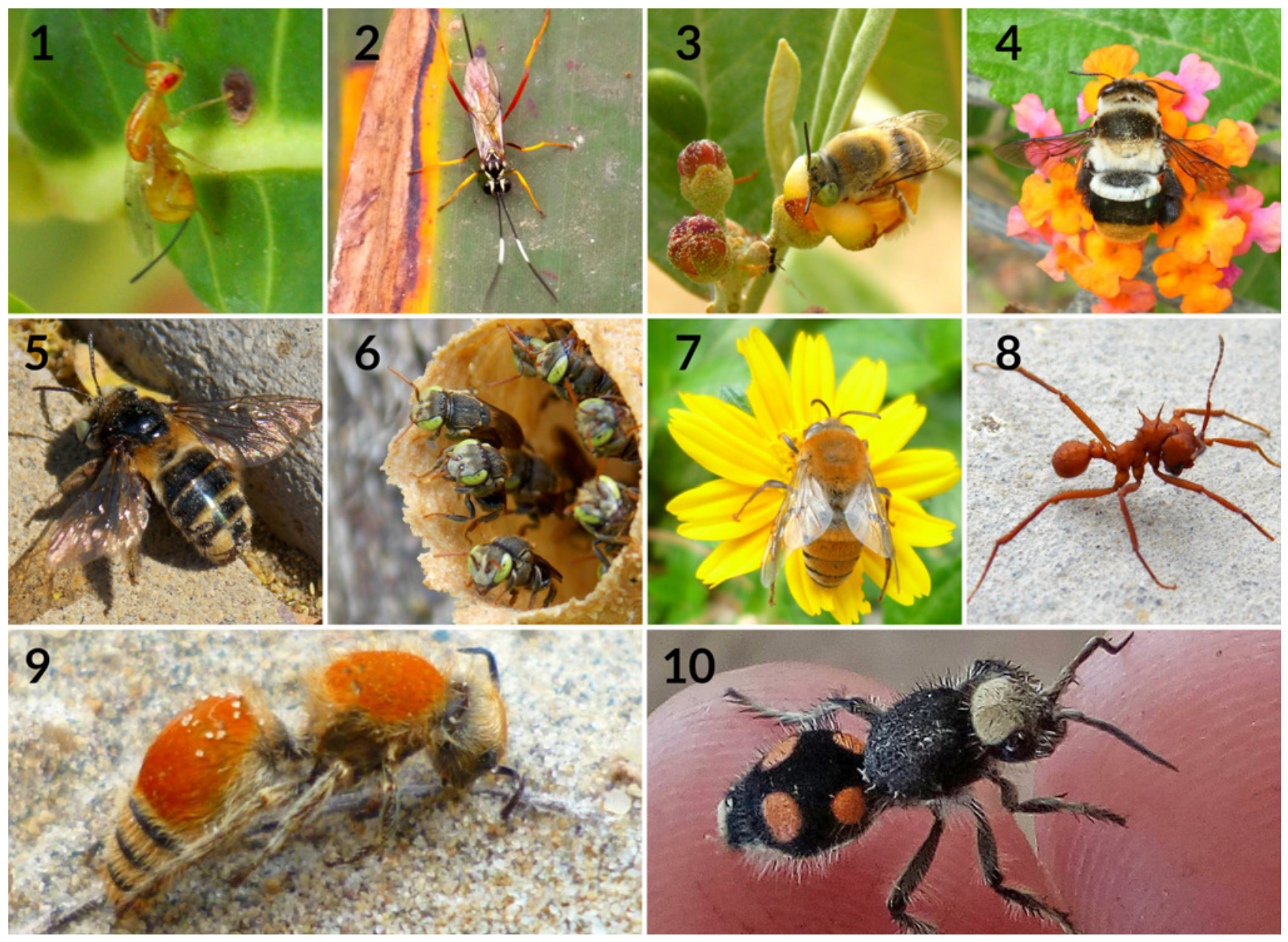

Figs. 1-10.- Habitus. 1. Megastigmus transvaalensis (Hussey, 1956). 2. Cryptanura sp. 3. Centris (Penthemisia) mixta Friese, 1904. 4. Centris (Centris) maculifrons Smith, 1854. 5. Florilegus (Florilegus) purpurascens Cockerell, 1914. 6. Nannotrigona mellaria (Smith, 1862). 7. Caupolicana piurensis Cockerell, 1911. 8. Acromyrmex subterraneus peruvianus Borgmeier, 1940.9. Quwitilla blattoserica (Kohl, 1882). 10. Traumatomutilla vitelligera (Gerstäcker, 1874) [Fotografías 1-9 por G. Juárez; Fotografía 10 por I. Caballero].

Figs. 1-10.- Habitus. 1. Megastigmus transvaalensis (Hussey, 1956). 2. Cryptanura sp. 3. Centris (Penthemisia) mixta Friese, 1904. 4. Centris (Centris) maculifrons Smith, 1854. 5. Florilegus (Florilegus) purpurascens Cockerell, 1914. 6. Nannotrigona mellaria (Smith, 1862). 7. Caupolicana piurensis Cockerell, 1911. 8. Acromyrmex subterraneus peruvianus Borgmeier, 1940. 9. Quwitilla blattoserica (Kohl, 1882). 10. Traumatomutilla vitelligera (Gerstäcker, 1874) [Photographs 1-9 by G. Juárez; Photographs 10 by I. Caballero].

REFERENCIA. Moure \& Dal Molin (2012).

Familia Apidae Latreille, 1802

Subfamilia Apinae Latreille, 1802

Género Centris Fabricius, 1804

34. Centris (Penthemisia) mixta Friese, 1904 (Fig. 3)

Material examinado. PERÚ. 1 , Morropón, Piedra del Toro, $05^{\circ} 11^{\prime} \mathrm{S}-80^{\circ} 49^{\prime} \mathrm{O}, 1.225 \mathrm{msnm}, 22-\mathrm{XI}-2019$, red entomológica, G. Juárez, Bosque estacionalmente seco de colina; 1 ㅇ, Morropón, $05^{\circ} 05^{\prime} \mathrm{S}-80^{\circ} 99^{\prime} \mathrm{O}, 1.225 \mathrm{msnm}, 19-\mathrm{XII}-2019$, red entomológica, U. González, Bosque estacionalmente seco de colina; 1 ㅇ, Morropón, Buenos Aires, $05^{\circ} 05^{\prime} 51^{\prime \prime} \mathrm{S}-80^{\circ} 09^{\prime} 44^{\prime \prime} \mathrm{O}$, $1.225 \mathrm{msnm}, 13-\mathrm{X}-2019$, red entomológica, U. González, Bosque estacionalmente seco de colina; 1 , Ayabaca, Suyo, $04^{\circ} 30^{\prime} 45^{\prime \prime} \mathrm{S}-80^{\circ} 02^{\prime} 10^{\prime \prime} \mathrm{O}, 300 \mathrm{msnm}$, 07-I-2020, red entomológica, G. Juárez, Bosque estacionalmente seco de colina.

Comentario. Especie que se distribuye en Chile y Perú y que se caracteriza por habitar ecosistemas secos y desérticos (Vieira-
Zanella, 2002; Moure \& Dal Molin, 2012; Juárez-Noé \& González-Coronado, 2018). Especie que amplía su área de distribución conocida dentro de la región a las provincias de Morropón y Ayabaca, anteriormente registrada para las provincias de Piura, Sullana, Talara, Paita, Sechura. Los individuos examinados se colectaron sobre hojas y flores de Capparis avicennifolia Kunth (Capparaceae).

35. Centris (Centris) maculifrons Smith, 1854 (Fig. 4)

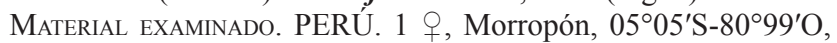
1.225 msnm, 19-XII-2019, red entomológica, U. González, Bosque estacionalmente seco de colina; $1 \hat{\delta}$, Ayabaca, Suyo, $04^{\circ} 30^{\prime} 45^{\prime \prime} \mathrm{S}-80^{\circ} 02^{\prime} 10^{\prime \prime} \mathrm{O}, 300 \mathrm{msnm}, 07-\mathrm{I}-2020$, red entomológica, G. Juárez, Bosque estacionalmente seco de colina.

Comentario. Especie que se distribuye en Brasil, Colombia, Ecuador y Perú (Moure \& Dal Molin, 2012). Hasta el momento esta especie se registra en ecosistemas secos y desérticos de la región Piura (Juárez-Noé \& González-Coronado, 2018). 
Especie que amplía su área de distribución conocida dentro de la región a las provincias de Morropón y Ayabaca, anteriormente registrada para las provincias de Piura, Sullana, Talara, Paita, Sechura. Los individuos examinados se colectaron en Lantana sp (Verbenaceae).

Género Florilegus Robertson, 1900.

36. Florilegus (Florilegus) purpurascens Cockerell, 1914 (Fig. 5) Material examinado. PERÚ. 1 \% , Morropón, $05^{\circ} 05^{\prime} \mathrm{S}-80^{\circ} 99^{\prime} \mathrm{O}$, 1.225 msnm, 19-XII-2019, red entomológica, U. González, Bosque estacionalmente seco de colina; $1 \hat{\delta}$, Ayabaca, Suyo, $04^{\circ} 30^{\prime} 45^{\prime \prime} \mathrm{S}-80^{\circ} 02^{\prime} 10^{\prime \prime} \mathrm{O}, 300 \mathrm{msnm}, 07-\mathrm{I}-2020$, red entomológica, G. Juárez, Bosque estacionalmente seco de colina.

Comentario. Especie que se distribuye en Ecuador y Perú (Moure \& Dal Molin, 2012) y que hasta el momento se registra en ecosistemas secos y desérticos de la región Piura (Juárez-Noé \& González-Coronado, 2018). Especie que amplía su área de distribución conocida dentro de la región a las provincias de Morropón y Ayabaca, anteriormente registrada para las provincias de Piura, Sullana, Talara, Paita, Sechura, Talara. Los individuos examinados se colectaron posadas a ras de suelo en áreas desprovistas de vegetación.

Género Nannotrigona Cockerell, 1922

37. Nannotrigona mellaria (Smith, 1862) (Fig. 6)

Material examinado. PERÚ. 3 ठิ $\widehat{\partial}$, Ayabaca, Suyo, $04^{\circ} 30^{\prime} 45^{\prime \prime} \mathrm{S}-80^{\circ} 02^{\prime} 10^{\prime \prime} \mathrm{O}, \quad 300 \mathrm{msnm}, 07-\mathrm{I}-2020$, red entomológica, G. Juárez, Bosque estacionalmente seco de colina; 2 우우, Huancabamba, $05^{\circ} 27^{\prime} 07^{\prime \prime} \mathrm{S}-79^{\circ} 36^{\prime} 22^{\prime \prime} \mathrm{O}$, 300 msnm, 15-VIII-2019, trampa de intercepción, G. Juárez, Bosque estacionalmente seco de colina.

Comentario. Especie de distribución neotropical registrada en Costa Rica, Nicaragua, Panamá, Ecuador, Colombia y Perú (Rasmussen \& González, 2017). En la región Piura se encuentra en ecosistemas secos y desérticos y asociada a $P$. pallida donde construye sus nidos y visita sus flores (Juárez-Noé \& González-Coronado, 2018; 2020). Especie que amplía su área de distribución conocida dentro de la región a las provincias de Ayabaca y Huancabamba, anteriormente registrada para las provincias de Piura, Sullana, Talara, Paita, Sechura, Morropón (Juárez-Noé \& González-Coronado, 2018). Los individuos examinados se colectaron sobre inflorescencias de P. pallida.

Familia Colletidae Lepeletier, 1841

Subfamilia Diphaglossinae Vachal, 1909

Género Caupolicana Spinola, 1851

38. Caupolicana piurensis Cockerell, 1911* (Fig. 7)

Material examinado. PeRÚ. 1 \%, Morropón, $05^{\circ} 05^{\prime} \mathrm{S}-80^{\circ} 99^{\prime} \mathrm{O}$, $1.225 \mathrm{msnm}, 15-\mathrm{X}-2019$, trampa de intercepción, G. Juárez, Bosque estacionalmente seco de colina; $2 \hat{\delta} \hat{\partial}$, Sechura, Sechura, $05^{\circ} 34^{\prime} \mathrm{S}-81^{\circ} 16^{\prime} \mathrm{O}, 16 \mathrm{msnm}, 16-\mathrm{IX}-2019$, red entomológica, G. Juárez, Bosque estacionalmente seco de llanura; 2 오, Paita, Paita, $05^{\circ} 04^{\prime} \mathrm{S}-81^{\circ} 06^{\prime} \mathrm{O}, 10 \mathrm{msnm}, 15-\mathrm{V}-$ 2019, red entomológica, U. González, Bosque estacionalmente seco de llanura.

Comentario. Especie que hasta el momento se registra en ecosistemas secos y desérticos de la región Piura (Juárez-Noé \& González-Coronado, 2018). Especie que amplía su área de distribución conocida dentro de la región a las provincias de Paita, Sechura, Morropón, anteriormente registrada para las provincias Piura, Sullana, Talara (Juárez-Noé \& González-Coronado, 2018). Los individuos examinados se colectaron sobre flores de Helianthus annuus L. (Asteraceae) y C. avicennifolia (Capparaceae).
Familia Crabronidae Latreille, 1802

Subfamilia Bembecinae Latreille, 1802

Género Trichostictia J. Parker, 1929

39. Trichostictia brunneri J. Parker, 1929

Material examinado. PERÚ. 1 q, Morropón, $05^{\circ} 05^{\prime} \mathrm{S}-80^{\circ} 99^{\prime} \mathrm{O}$, 1.225 msnm, 19-II-2019, red entomológica, U. González, Bosque estacionalmente seco de colina; 1 \&, Morropón, Piedra del Toro, $05^{\circ} 11^{\prime} \mathrm{S}-80^{\circ} 49^{\prime} \mathrm{O}, 1.225 \mathrm{msnm}$, 19-II-2019, red entomológica, G. Juárez, Bosque estacionalmente seco de colina; 2 우우, Morropón, Buenos Aires, $05^{\circ} 05^{\prime} 51^{\prime \prime} \mathrm{S}-80^{\circ} 09^{\prime} 44^{\prime \prime} \mathrm{O}$, 1.225 msnm, 19-II-2019, red entomológica, U. González, Bosque estacionalmente seco de colina.

Comentario. Especie cuya distribución abarca Perú y Chile y que se caracteriza por habitar en áreas secas y desérticas (Sielfeld, 1980; Rasmussen \& Asenjo, 2009; Juárez-Noé \& GonzálezCoronado, 2018). Especie que amplía su área de distribución conocida dentro de la región a la provincia de Morropón, anteriormente registrada para las provincias de Talara, Sullana (Juárez-Noé \& González-Coronado, 2018). Los individuos examinados se colectaron a ras del suelo en áreas desprovistas de vegetación y visitando flores de Colicodendron scabridum (Kunth) Seemann y C. avicennifolia (Capparaceae).

Familia Megachilidae Latreille, 1802

Subfamilia Megachilinae Latreille, 1802

Género Megachile Latreille, 1802

40. Megachile (Austromegachile) philinca Cockerell, $1912+$

Material examinado. PERÚ. Piura, 05¹0'51"S-80³7'31"O, $29 \mathrm{msnm}$, Bosque estacionalmente seco de llanura.

Comentario. Especie cuya distribución abarca Ecuador y Perú (Raw, 2002; Moure \& Dal Molin, 2012).

ReFerencia. Moure \& Dal Molin (2012).

41. Megachile (Dasymegachile) piurensis Cockerell, $1911+*$ Material examinado. Perú. Piura, $05^{\circ} 10^{\prime} 51^{\prime \prime} \mathrm{S}-80^{\circ} 37^{\prime} 31^{\prime \prime} \mathrm{O}$, 29 msnm, Bosque estacionalmente seco de llanura.

Comentario. Especie conocida solamente de Perú específicamente desde la región Piura (Moure \& Dal Molin, 2012).

ReFEREnCIA. Moure \& Dal Molin (2012).

Superfamilia Vespoidea Latreille, 1802

Familia Formicidae Latreille, 1809

Subfamilia Myrmicinae Lepeletier de Saint-Fargeau, 1835

Género Acromyrmex Mayr, 1865

42. Acromyrmex subterraneus peruvianus Borgmeier, 1940 (Fig. 8)

Material examinado. PERÚ. 1 q, Huancabamba, Canchaque, Cerro Huayanay, $05^{\circ} 24^{\prime} 07^{\prime \prime S}-79^{\circ} 36^{\prime} 22^{\prime \prime} \mathrm{O}, 1.600 \mathrm{msnm}, 16-\mathrm{I}-$ 2020, colecta directa, U. González, Bosque seco interandino, FORM015-GJN; 1 गे, Huancabamba, Canchaque, Pampa Minas, $05^{\circ} 21^{\prime} 20.6^{\prime \prime} \mathrm{S}-79^{\circ} 35^{\prime} 20^{\prime \prime} \mathrm{O}, 2.100 \mathrm{msnm}, 16-\mathrm{I}-2020$, trampa pitfall, U. González, Bosque seco interandino,

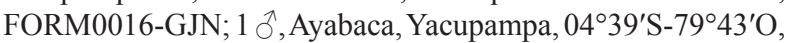
2.755 msnm, 04-XI-2019, trampa pitfall, G. Juárez, Bosque húmedo de montaña, FORM0019-GJN.

Comentario. Mencionada como Acromyrmex sp en Juárez-Noé \& González-Coronado (2018). El género Acromyrmex tiene distribución netamente neotropical (Fernández, 2003). Especie que se distribuye en ecosistemas andinos de la región Piura (Juárez-Noé \& González-Coronado, 2018). Los individuos examinados se colectaron a ras del suelo en áreas de bosque con abundante vegetación.

Subfamilia Dorylinae Leach, 1815

Género Eciton Latreille, 1804

43. Eciton $\mathrm{sp}$ 
Material examinado. PeRÚ. 1 q, Huancabamba, Canchaque, Cerro Huayanay, $05^{\circ} 24^{\prime} 07^{\prime \prime} \mathrm{S}-79^{\circ} 36^{\prime} 22^{\prime \prime} \mathrm{O}, 1.600 \mathrm{msnm}$, 10-II-2020, trampa pitfall, U. González, Bosque seco interandino; 1 ô, Huancabamba, Canchaque, Pampa Minas, $05^{\circ} 21^{\prime} 20.6^{\prime \prime} \mathrm{S}-79^{\circ} 35^{\prime} 20^{\prime \prime} \mathrm{O}, 2.100 \mathrm{msnm}$, 11-II-2020, colecta directa, G. Juárez, Bosque seco interandino; 1 ઈ, Ayabaca, Yacupampa, $04^{\circ} 39^{\prime} \mathrm{S}-79^{\circ} 43^{\prime} \mathrm{O}, 2.755 \mathrm{msnm}, 21-\mathrm{I}-2020$, trampa pitfall, G. Juárez, Bosque húmedo de montaña, FORM0019GJN.

COMEnTARio. El género Eciton es de distribución netamente neotropical (Palacio, 2003). Especie que se distribuye en ecosistemas andinos de la región Piura (Juárez-Noé \& González-Coronado, 2018). Los individuos examinados se colectaron a ras del suelo y entre la hojarasca en áreas de bosque con abundante vegetación.

Familia Mutillidae Latreille, 1802

Subfamilia Sphaeropthalminae Schuster, 1949

Género Quwitilla Williams, Bartholomay \& Cambra, 2019

Comentario. El género Dasymutilla Ashmead 1899 contiene 11 especies distribuidas en Sur América (Williams, 2012; Bartholomay et al., 2019). Recientemente Bartholomay et al. (2019) realizaron una revisión de este género en la cual cuatro especies pasaron a formar parte del nuevo género Quwitilla.

44. Quwitilla blattoserica (Kohl, 1882) (Fig. 9)

Material eXaminado. PERÚ. 1 o, Morropón, $05^{\circ} 05^{\prime} \mathrm{S}-80^{\circ} 99^{\prime} \mathrm{O}$, 1.225 msnm, 09-IX-2019, colecta manual, U. González, Bosque estacionalmente seco de colina; 1 , Morropón, Piedra del Toro, $05^{\circ} 11^{\prime} \mathrm{S}-80^{\circ} 49^{\prime} \mathrm{O}, 1.225 \mathrm{msnm}$, 09-IX-2019, trampa pitfall, G. Juárez, Bosque estacionalmente seco de colina; 2 oे $^{\circ}$, Ayabaca, Suyo, 04 $30^{\circ} 45^{\prime \prime} \mathrm{S}-80^{\circ} 02^{\prime} 10^{\prime \prime} \mathrm{O}, 300 \mathrm{msnm}$, 07-I-2020, trampa pitfall, G. Juárez, Bosque estacionalmente seco de colina.

Comentario. Mencionada como Dasymutilla blattoserica (Kohl, 1882) en Juárez-Noé \& González-Coronado (2018). Se distribuye en Chile y Perú (Bartholomay et al., 2019). Hasta el momento para la región Piura se registra en ecosistemas de bosque seco y desierto costero (Juárez-Noé \& GonzálezCoronado, 2018). Especie que amplía su área de distribución dentro de la región a las provincias de Morropón y Ayabaca, anteriormente registrada para las provincias de Sullana, Piura, Talara, Paita, Sechura (Juárez-Noé \& González-Coronado, 2018). Los individuos examinados se colectaron en áreas de bosques desprovistas de vegetación.

\section{Quwitilla peruviana (Suárez, 1970)}

Material examinado. PERÚ. 1 q, Talara, Los Órganos, $04^{\circ} 10^{\prime} 38^{\prime \prime} \mathrm{S}-81^{\circ} 07^{\prime} 27^{\prime \prime} \mathrm{O}, 32 \mathrm{msnm}, 22-\mathrm{I}-2020$, colecta manual, G. Juárez, Bosque estacionalmente seco de llanura; 1 ô, Sechura, Sechura, $05^{\circ} 34^{\prime} \mathrm{S}-81^{\circ} 16^{\prime} \mathrm{O}, 16 \mathrm{msnm}, 216-\mathrm{X}-2019$, trampa pitfall, G. Juárez, Bosque estacionalmente seco de llanura.

Comentario. Mencionada como Dasymutilla peruviana Suárez, 1970 en Juárez-Noé \& González-Coronado (2018). Especie conocida solamente de Perú (Bartholomay et al., 2019). Reciente Dasymutilla homochroma Suárez, 1970, especie listada en Juárez-Noé \& González-Coronado (2018), fue considerada como sinónimo de $Q$. peruviana por Bartholomay et al. (2019). Especie que amplía su área de distribución dentro de la región a las provincias de Talara y Sechura, anteriormente registrada para la provincia de Piura. Los individuos examinados se colectaron en áreas de bosques desprovistas de vegetación.

Género Traumatomutilla André, 1901

46. Traumatomutilla vitelligera (Gerstäcker, 1874) (Fig. 10)
Material EXAMINADO. PERÚ. Talara, Lobitos, $04^{\circ} 27^{\prime} 10^{\prime \prime} \mathrm{S}-81^{\circ} 16^{\prime} 40^{\prime \prime} \mathrm{O}, 12 \mathrm{msnm}, 27-\mathrm{XI}-2019$, colecta manual, G. Juárez, Bosque estacionalmente seco de llanura; 1 \&, Talara, El Alto, $04^{\circ} 16^{\prime} 04^{\prime \prime} \mathrm{S}-81^{\circ} 13^{\prime} 09^{\prime \prime} \mathrm{O}, 300 \mathrm{msnm}, 01-\mathrm{X}-$ 2019, colecta manual, G. Juárez, Bosque estacionalmente seco de colina; 1 \&, Paita, Paita, $05^{\circ} 04^{\prime} \mathrm{S}-81^{\circ} 06^{\prime} \mathrm{O}, 10 \mathrm{msnm}, 15-$ XI-2019, trampa pitfall, U. González, Bosque estacionalmente

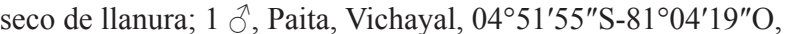
10 msnm, 1-XI-2019, trampa pitfall, G. Juárez, Bosque estacionalmente seco de llanura; 2 के $\hat{\sigma}^{2}$ Sechura, Sechura, $05^{\circ} 34^{\prime} \mathrm{S}-81^{\circ} 16^{\prime} \mathrm{O}, 10 \mathrm{msnm}, 17-\mathrm{XI}-2019$, trampa pitfall, G. Juárez, Bosque estacionalmente seco de llanura; 1 , Morropón, $05^{\circ} 05^{\prime} \mathrm{S}-80^{\circ} 99^{\prime} \mathrm{O}, 1.225 \mathrm{msnm}, 09-\mathrm{IX}-2019$, colecta manual, U. González, Bosque estacionalmente seco de colina; $1 \delta^{\prime}$, Ayabaca, Suyo, 04 $30^{\prime} 45^{\prime \prime} \mathrm{S}-80^{\circ} 02^{\prime} 10^{\prime \prime} \mathrm{O}, 300 \mathrm{msnm}$, 07-I-2020, trampa pitfall, G. Juárez, Bosque estacionalmente seco de colina; 1 을 Huancabamba, $05^{\circ} 27^{\prime} 07^{\prime \prime} \mathrm{S}-79^{\circ} 36^{\prime} 22^{\prime \prime} \mathrm{O}$, 300 msnm, 15-VIII-2019, colecta manual, G. Juárez, Bosque estacionalmente seco de colina.

Comentario. Se distribuye en zonas áridas y semiáridas de Ecuador y Perú (Williams et al., 2017; Juárez \& Caballero, 2018). Hasta el momento para la región Piura se registra en ecosistemas de bosques secos y desierto costero (JuárezNoé \& González-Coronado, 2018). Especie que amplía su área de distribución dentro de la región a las provincias de Sechura, Morropón, Paita, Talara, Ayabaca y Huancabamba, anteriormente registrada para las provincias de Piura, Sullana (Juárez-Noé \& González-Coronado, 2018). Los individuos examinados se colectaron en áreas de bosques desprovistas de vegetación.

Género Pseudomethoca Ashmead, 1896

47. Pseudomethoca piura (Casal, 1970)

Material eXAminado. PERÚ. Talara, Lobitos,

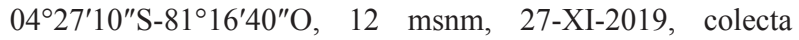
manual, G. Juárez, Bosque estacionalmente seco de llanura; 1 ㅇ, Talara, El Alto, $04^{\circ} 16^{\prime} 04^{\prime \prime} \mathrm{S}-81^{\circ} 13^{\prime} 09^{\prime \prime} \mathrm{O}, 300 \mathrm{msnm}, 01-\mathrm{X}-$ 2019, colecta manual, G. Juárez, Bosque estacionalmente seco de colina; 1 ㅇ, Morropón, $05^{\circ} 05^{\prime} \mathrm{S}-80^{\circ} 99^{\prime} \mathrm{O}, 1.225 \mathrm{msnm}, 09$ IX-2019, trampa pitfall, U. González, Bosque estacionalmente seco de colina.

Comentario. Esta especie para la región Piura se distribuye hasta el momento en ecosistemas de bosques secos y desierto costero (Quintero \& Cambra, 1996; Juárez-Noé \& González-Coronado, 2018). Especie que amplía su área de distribución dentro de la región a las provincias de Talara y Morropón, anteriormente registrada para las provincias de Piura, Sullana, Paita, Sechura (Juárez-Noé \& González-Coronado, 2018). Los individuos examinados se colectaron en áreas de bosques desprovistas de vegetación.

Familia Vespidae Leach, 1815

Subfamilia Eumeninae Leach, 1815

Género Ancistroceroides Saussure, 1855

48. Ancistroceroides $\mathrm{sp}$

Material examinado. PeRÚ. 1 q, Talara, El Alto, $04^{\circ} 16^{\prime} 04^{\prime \prime} \mathrm{S}-81^{\circ} 13^{\prime} 09^{\prime \prime} \mathrm{O}, \quad 300 \mathrm{msnm}, 11-\mathrm{X}-2019$, red entomológica, G. Juárez, Bosque estacionalmente seco de colina.

Comentario. La distribución del género Ancistroceroides abarca la región neártica y neotropical (Carpenter \& Garcete-Barrett, 2002; Carpenter, 2004). El individuo examinado fue colectado sobre hojas de C. scabridum.

Género Parancistrocerus Bequaert, 1925

49. Parancistrocerus sp 
Material examinado. Perú. 1 , Talara, El Alto, 04 $16^{\prime} 04^{\prime \prime} \mathrm{S}-81^{\circ} 13^{\prime} 09^{\prime \prime} \mathrm{O}, \quad 300 \mathrm{msnm}, 11-\mathrm{X}-2019$, red entomológica, G. Juárez, Bosque estacionalmente seco de colina.

Comentario. La distribución del género Parancistrocerus abarca las regiones neártica, neotropical y paleártica oriental (Carpenter \& Garcete-Barrett, 2002). El individuo examinado se colectó sobre hojas de C. scabridum.

\section{Agradecimientos}

A Bolívar Garcete-Barrett (Chalcididae, Crabronidae, Ichneumonidae, Pompilidae, Sphecidae, Vespidae), Roberto Barrera (Vespidae), Kevin Williams (Mutillidae), Claus Rasmussen, John Ascher (Apidae), Felipe Vivallo (Apidae, Halictidae, Megachilidae) y James C. Trager (Formicidae) por el envío de material bibliográfico y ayuda en la identificación de algunas especies. A los revisores del manuscrito por sus sugerencias.

\section{Referencias}

Aliaga, J., 2012. Presencia de Trialeurodes variabilis (Quaintance, 1900) y su parasitoide Eretmocerus eremicus Rose \& Zolnerowich en cultivos de yuca Manihot esculenta Crantz en Supe - Barranca, LimaPerú. Revista Peruana de Entomología, 47(1): 12-14.

Astola-Mariscal, S. Z. \& Narrea-Cango, M., 2019. Biología y comportamiento de Cotesia flavipes Cameron (Braconidae) parasitoide de Diatraea saccharalis Fabricius (Crambidae). Ecología Aplicada, 18(1): 77-83. https:// doi.org/10.21704/rea.v18i1.1309

Bartholomay, P. R., Williams, K. A. Cambra, R. A. \& Oliveira, M. L., 2019. Does the genus Dasymutilla Ashmead occur in South America? The new genus Quwitilla, new combinations, and new distribution records from Neotropical velvet ants (Hymenoptera: Mutillidae). Zootaxa, 4623(2): 261-281. https://doi. org/10.11646/zootaxa.4623.2.3

Bezděčková, K., Bezdecka, P. \& Machar, I., 2015. A Checklist of the ants (Hymenoptera: Formicidae) of Peru. Zootaxa, 4020(1): 101-133. https://doi. org/10.11646/zootaxa.4020.1.4

Beingolea, O., 1959. El problema de la mosca blanca lanuda de los cítricos en el Perú Aleurothrixus floccosus (Homoptera: Aleurodidae). Revista Peruana de Entomología Agrícola, 2(1): 65-68.

Beingolea, O., 1967. Control biológico de las plagas de los cítricos en el Perú. Revista Peruana de Entomología, 10(1): 67-81.

Beingolea, O., 1969. Notas sobre la biología de Selenaspidus articulatus Morgan (Hom.: Diaspididae), "queresa redonda de los cítricos". Revista Peruana de Entomología, 12(1): 119-129.

Buffington, M. L., 2010. A revision of Ganaspidium Weld, 1952 (Hymenoptera, Figitidae, Eucoilinae): new species, bionomics, and distribution. ZooKeys, 37: 81101. https://doi.org/10.3897/zookeys.37.311

Cardoso da Costa-Lima, T., Mendonca das Chagas, M. C. \& Pastoli Parra, J. R., 2014. Temperaturedependent development of two neotropical parasitoids of Liriomyza sativae (Diptera: Agromyzidae). Journal of Insect Science, 14(25): 1-4. https://doi.org/10.1093/ jisesa/ieu107

Carrasco, F., 1972. Catálogo de la familia Ichneumonidae peruanos. Revista Peruana de Entomología, 15(2): 324332.

Carpenter, J. \& Garcete-Barrett, B., 2002. A key to the neotropical genera of Eumeninae (Hymenoptera: Vespidae). Boletín del Museo Nacional Historia Natural Paraguay, 14(1-2): 52-73.

Carpenter, J., 2004. Ancistroceroides de Saussure, a potter wasp genus new for the United States, with a new key to the genera of Eumeninae of America North of Mexico (Hymenoptera: Vespidae). Journal of the Kansas Entomology, 77(4): 721-741. https://doi. org/10.2317/E-33.1

Castillo-Carrillo, P., Elizalde, R. \& Rasmussen, C., 2013. Inventario de las abejas nativas sin aguijón (Hymenoptera: Apidae: Meliponini) en Tumbes-Perú. Revista Notas Apícolas, 16: 43-50.

Cueva, M. 1980. Diatraea saccharalis (Fab.) y sus factores bióticos de mortalidad natural durante el periodo vegetativo de la caña de azúcar. Revista Peruana de Entomología, 23(1): 77-81.

Da Silva Dias, N., Broglio-Micheletti, S. Moraes Farias, N. Silva da Costa, S. dos Santos, J. Pexioto Lopes, D. \& Costa, V. 2011. Ocorrência de Telenomus alecto Crawford, 1914 (Hymenoptera: Scelionidae) em ovos de Diatraea spp. (Lepidoptera: Crambidae) em cana-de-açúcar no estado de Alagoas, Brasil. IDESIA (Chile), 29(3): 95-97. https://doi.org/10.4067/S071834292011000300014

Dos Santos, E., Campanholo-Grandinete, Y. \& BarbosaNoll, F., 2015. Additions to the checklist of Scoliidae, Sphecidae, Pompilidae and Vespidae of Peru, with notes on the endemic status of some species (Hymenoptera, Aculeata).ZooKeys, 519: 33-48. https://doi.org/10.3897/ zookeys.519.6501

Delvare, G. \& Huchet, J. 2017. Brachymeria mochica, a new Neotropical species of Chalcididae (Hymenoptera: Chalcidoidea) discovered on the archaeological site of Huacas de Moche, Peru with a review of related species. Zootaxa, 4290(1): 43-60. https://doi.org/10.11646/ zootaxa.4290.1.2

Escalante, J., 1991. Especies de hormigas conocidas de Perú (Hymenoptera: Formicidae). Revista Peruana de Entomología, 34: 1-13.

Farhad, A., Talebi, A. A. Fathipour, Y. Hajiqanbar, H. R. \& Strumia, F., 2017. The genus Holopyga (Hymenoptera: Chrysididae) in Iran, with five new records. Journal of Agricultural Science and Technology, 19: 877-888.

Fernández, F., 2003. Introducción a las hormigas de la región Neotropical. Instituto de Investigación de Recursos Biológicos Alexander von Humboldt, Bogotá, Colombia, 398 pp.

Fernández, F. \& Sharkey, M., 2006. Introducción a los Hymenoptera de la Región Neotropical. Sociedad Colombiana de Entomología, Bogotá D.C, 894 pp.

Fernández, S.\& Pujade-Villar, J., 2015. Orden Hymenoptera. RevistaIde@-SEA, 59: 1-36. 
Fernandes, D. R. R., Salas, C. Rothmann, S. Lara, R. I. R. \& Perioto, N. W., 2014. Megastigmus transvaalensis (Hymenoptera: Torymidae) on Schinus polygamus (Anacardiaceae): a new native host for this invasive seed-feeding species. IDESIA (Chile), 32(4): 119-121. https://doi.org/10.4067/S0718-34292014000400016

Fujie, S., Shimizu, S. \& Fernández-Triana, J., 2018. A new species and a key to world species of the flavipes species-group of the genus Cotesia 1 Cameron, 1891 (Hymenoptera: Braconidae: Microgastrinae) from Japan. Zootaxa, 4527: 372-380. https://oi. org/10.11646/zootaxa.4527.3.6

García, J., 2003. Comparación de la captura de Hymenoptera (Insecta) mediante cuatro métodos de muestreo, en los cerros Yaví y Yutajé del Pantepui venezolano. Entomotropica, 18(1): 27-35.

Giraldo, A. \& Arellano, G., 2003. Resiliencia de la comunidad epígea de Coleoptera en las Lomas de Lachay después del evento El Niño 1997-98. Ecología Aplicada, 2(1): 5968. https://doi.org/10.21704/rea.v2i1-2.248

Gómez de Picho, H., 1987. Biología de Telenomus remus Nixon (Hymenoptera: Scelionidae). Revista Peruana de Entomología, 30: 29-32.

Grissell, E. E. \& Goodpasture, C. E., 1981. A review of nearctic Podagrionini, with description of sexual behavior of Podagrion mantis (Hymenoptera: Torymidae). Annals of the Entomological Society of America, 74: 226-241. https://doi.org/10.1093/ aesa/74.2.226

Guénard, B. \& Economo, E. P., 2015. Additions to the checklist of the ants (Hymenoptera: Formicidae) of Peru. Zootaxa, 4040(2): 225-235. https://doi.org/10.11646/ zootaxa.4040.2.8

Hansson, C., 1995. Revised keys to the Nearctic species of Chrysocharis Föster (Hymenoptera: Eulophidae), including three new species. Journal of Hymenoptera Research, 4: 80-98.

Herrera, J. \& Álvarez, S., 1979. El control biológico de Bucculatrix thurberiella Busck (Lepidoptera: Lyonettidae) en Piura y Chira. Revista Peruana de Entomología, 22(1): 37-41.

Juárez, G. \& Caballero, C. I., 2018. Nuevo registro de dos especies de Mutillidae (Hymenoptera) en Perú. Boletín de la Sociedad Entomológica Aragonesa (S.E.A.), 62: 301-302.

Juárez-Noé, G. \& González-Coronado, U., 2018. Listado de himenópteros (Insecta: Hymenoptera) de la región Piura, Perú. Folia Entomológica Mexicana (nueva serie), 4(2): 48-65.

Juárez-Noé, G. \& González-Coronado, U., 2020. Lista taxonómica actualizada de los insectos asociados a Prosopis pallida (Humb. \& Bonpl. ex. Wild.) Kunth (Fabaceae) de la región Piura, Perú. Graellsia, 76(2): e110 [1-57]. https://doi.org/10.3989/graellsia.2020. v76.263

Kasparyan, D. R. \& Ruíz Cancino, E., 2006. Review of Mexican species of the genus Cryptanura Brullé with a key to species from North America (Hymenoptera: Ichneumonidae: Cryptini). Zoosystematica Rossica, 15(1): 87-112. https://doi.org/10.31610/zsr/2006.15.1.87
Kroschel, J., Mujica, N. Carhuapoma, P. \& Sporleder, M., 2016. Pest distribution and Risk Atlas for Africa. Available at https://cipotato.org/riskatlasforafrica/ (consultado 4.04.2020).

Masner, L., 1976. Revisionary notes and keys to world genera of Scelionidae (Hymenoptera: Proctotrupoidea). Memoirs of the Entomological Society of Canada, 97: 1-87. https://doi.org/10.4039/entm10897fv

Ministerio Del Ambiente., 2018. Mapa Nacional de Ecosistemas del Perú. Memoria Descriptiva. Ministerio del Ambiente (MINAM). Lima-Perú. 60 pp.

More, A., Villegas, P. \& Alzamora, M., 2014. Piura, Áreas prioritarias para la conservación de la biodiversidad. Naturaleza y Cultura Internacional-PROFONANPE. Lima. 166 pp.

Myartseva, S. \& Evans, G., 2007. Genus Encarsia Fórster of Mexico (Hymenoptera: Chalcidoidea: Aphelinidae). A revision, key and description of new species. Serie Avispas parasíticas de plagas y otros insectos. Universidad Autónoma de Tamaulipas. 323 pp.

Myartseva, S., Ruíz-Cancino, E. \& Coronado-Blanco, J. M., 2010. El género Aphytis Howard (Hymenoptera: Chalcidoidea: Aphelinidae) en México, clave de especies y descripción de una especie nueva. Dugesiana, 17(1): 81-94.

Myartseva, S., Ruíz-Cancino, E., Coronado-Blanco, J. M., Corona-López, A. M. \& Toledo-Hernández, V. H., 2012. Parasitoides (Hymenoptera: Aphelinidae, Signiphoridae, Platygastridae) de Aleurothrixus floccosus (Maskell, 1896) (Hemiptera: Aleyrodidae) en el estado de Veracruz, México, y descripción del macho de Encarsia dominicana Evans, 2002. Dugesiana, 19(1): 37-41.

Moure, J. S.\& Dal Molin, A., 2012. Catalogue of Bees (Hymenoptera, Apoidea) in the Neotropical Region online version. Available at http://moure.cria.org.br/ catalogue (Consultado 12.02.2020).

Mujica, N. \& Kroschel, J., 2011. Leafminer Fly (Diptera: Agromyzidae) occurrence, distribution, and Parasitoid associations in field and vegetable crops along the Peruvian Coast. Environmental Entomology, 40(2): 217-230. https://doi.org/10.1603/EN10170

Onody, H. C., Loffredo, A. P. S. \& Penteado-Dias, A. M., 2009. Notes on the Brazilian Brachycyrtus Kriechbaumer species (Hymenoptera, Ichneumonidae Brachycyrtinae). Brazilian Journal of Biology, 69(3): 981-982. https:// doi.org/10.1590/S1519-69842009000400030

Osorio-Mejía, P. A., 2018. Preferencia de Cotesia flavipes (Hymenoptera: Braconidae) sobre barrenadores Diatraea spp. (Lepidoptera: Crambidae) de caña para panela. Tesis para Magister en Ciencias Agrarias. Universidad Nacional de Colombia, 73 pp.

Palacio, E. E., 2003. Subfamilia Ecitoninae. En F. Fernández (ed.). Introducción a las hormigas de la región Neotropical. Instituto de Investigación de Recursos Biológicos Alexander von Humboldt, Bogotá, Colombia: 281-285.

Pardo, R., 1964. Clave para identificar los Formicidae de la provincia de Chiclayo. Revista Peruana de Entomología, 7(1): 98-102. 
Peters, R. S. \& Baur, H., 2011. A revision of the Dibrachys cavus species complex (Hymenoptera: Chalcidoidea: Pteromalidae). Zootaxa, 2937: 1-30. https://doi. org/10.11646/zootaxa.2937.1.1

Peters, R. S., Krogmann, L., Donath, A., Gunkel, S., Meusemann, K., Koslov, A., Podsiadlowski, L., Peterson, M., Lanfear, R., Diez, P. A., Heraty, J., Kjer, K. M., Klopfstein, S., Meier, R., Polidori, C., Schmitt, T., Liu, S., Zhou, X., Wappler, T., Rust, J., Misof, B. \& Niehuis, O., 2017. Evolutionary history of Hymenoptera. Current Biology, 27: 1-6. http://dx.doi.org/10.1016/j. cub.2017.01.027

Porter, Ch., 1985. Trachysphyrus and the new genus Aeliopotes in the Coastal Desert of Peru and north Chile (Hymenoptera: Ichneumonidae). Psyche, 92: 513-546.

Quintero, D. \& Cambra, R., 1996. Contribución a la sistemática de las mutílidas (Hymenoptera) del Perú, en especial las de la Estación Biológica BIOLAT, Río Manu, Pakitza. In Don E. Wilson \& A. Sandoval (eds.). Manu Biodiversity of Southeastern Peru. Smithsonian Institution Press, 679 pp.

Rasmussen, C., 2003. Clave de identificación para las especies peruanas de Bombus Latreille, 1809 (Hymenoptera, Apidae), con notas sobre su biología y distribución. Revista Peruana de Entomologia, 43: 31-45.

Rasmussen, C. \& Asenjo, A., 2009. A checklist to the wasps of Peru (Hymenoptera, Aculeata). ZooKeys, 15: 1-78. https://doi.org/10.3897/zookeys.15.196

Rasmussen, C. \& González, V., 2017. The neotropical stingless bee genus Nannotrigona Cockerell (Hymenoptera: Apidae: Meliponini): An illustrated key, notes on the types, and designation of lectotypes. Zootaxa, 4299(2): 191-220. https://doi.org/10.11646/ zootaxa.4299.2.2

Raw, A., 2002. New combinations and synonymies of leafcutter and mason bees of the Americas (Megachile, Hymenoptera, Megachilidae). Zootaxa, 71: 1-43. https://doi.org/10.11646/zootaxa.71.1.1

Redolfi de Huiza, I., 1994. Diversidad de Braconidae (Hymenoptera) en el Perú. Revista Peruana de Entomología, 37:11-22.

Reina, P. \& La Salle, J. 2003. Key to the World Genera of Eulophidae Parasitoids (Hymenoptera) of Leafmining Agromyzidae (Diptera). Available at https://keys. lucidcentral.org/keys/v3/eulophidae_parasitoids/

Rivera, J., 2003. Discovery of Podagrion brasiliense Howard, 1894 (Hymenoptera: Torymidae) as a parasitoid of the ootheca of Musonia surinama (Saussure, 1869) (Mantodea: Thespidae). Revista Peruana de Entomología, 43: 20.

Rodrigues-Lima, A., Jacobi, C. M. \& Fumi-Kumagai, A., 2012. A key to the Neotropical species of the Enicospilus ramidulus species-group (Hymenoptera: Ichneumonidae: Ophioninae), with the description of a new Brazilian species. Zootaxa, 3409: 63-68. https:// doi.org/10.11646/zootaxa.3409.1.5

Rodríguez-Berrío, A., 2006. Contribución al conocimiento de Enicospilus purgatus (Say, 1835) (Hymenoptera: Ichneumonidae). Revista Peruana de Entomología, 45: 125-126.
Rodríguez-Berrío, A., Bordera, S. \& Saaksjarvi, I. E., 2009. Checklist of Peruvian Ichneumonidae (Insecta, Hymenoptera). Zootaxa, 2303: 1-44. https://doi. org/10.11646/zootaxa.2303.1.1

Roques, A. \& Skrzypcznska, M., 2003. Seed-infesting chalcids of the genus Megastigmus Dalman, 1820 (Hymenoptera: Torymidae) native and introduced to the West Palaearctic region: taxonomy, host specificity and distribution. Journal of Natural History, 37: 127$238 \mathrm{https}: / /$ doi.org/10.1080/713834669

Ruz, L., 1991. Classification and phylogenetic relationships of the Panurgine Bees: the Calliopsini and allies (Hymenoptera: Andrenidae). The University of Kansas Science Bulletin, 54 (7): 209-256. https://doi. org/10.5962/bhl.part. 19640

Sarmiento, C. E., 2006. Métodos generales de recolección. In: Fernández, F. \& M. J. Sharkey (eds.). Introducción a los Hymenoptera de la Región Neotropical. Sociedad Colombiana de Entomología y Universidad Nacional de Colombia, Bogotá D. C.: 115-132.

Sharkey, M., 2007. Phylogeny and Classification of Hymenoptera. In Zhang, Z.-Q. \& W.A. Shear (eds.). Linnaeus Tercentenary: Progress in Invertebrate Taxonomy. Zootaxa, 1668: 521-548. https://doi. org/10.11646/zootaxa.1668.1.25

Sielfeld, W., 1980. Las especies de Sphecidae(Hymenoptera) conocidas para territorio chileno. Revista Chilena de Entomología, 10: 71-76.

Supanta-Huarcaya, L. M., 2017. La temperatura sobre la biología de Encarsia tabacivora Viggiani (Hymenoptera: Aphelinidae) parasitoide de Bemisia tabaci (Gennadius) (Hemiptera: Aleyrodidae). Tesis para Ingeniero Agrónomo. Universidad Nacional Agraria La Molina. $141 \mathrm{pp}$.

Urban, D. \& Moure, J. S., 2001. Catálogo de Apoidea da Região Neotropical (Hymenoptera, Colletidae). II. Diphaglossinae. Revista Brasileira de Zoologia, 18(1): 1-34. https://doi.org/10.1590/S0101-81752001000100001

Urban, D., 2003. Santiago wittmanni sp, nv. do Peru e notas sobre Eucerini (Hymenoptera: Anthophoridae. Revista Brasileira de Zoologia, 20(2): 201-205. https://doi. org/10.1590/S0101-81752003000200005

Velásquez de Ríos, M. \& Terán, J., 2003. Los Trichogramma (Hymenoptera: Trichogrammatidae) de la región noroccidental del estado Guárico, Venezuela. Entomotropica, 18(2): 127-145.

Vieira-Zanella, F. C., 2002. Sistemática, filogenia e distribuição geográfica das espécies sul-americanas de Centris (Paracentris) Cameron, 1903 e de Centris (Penthemisia) Moure, 1950, incluindo uma análise filogenética do "grupo Centris" sensu Ayala, 1998 (Hymenoptera, Apoidea, Centridini). Revista Brasileira de Entomologia, 46(4): 435-488. https://doi. org/10.1590/S0085-56262002000400001

Williams, K. A., 2012. Systematics of Mutillidae (Hymenoptera) with Special Emphasis on Dasymutilla and Their Allies. Thesis Doctor of Philosophy. Utah State University. 342 pp.

Williams, K., Bartholomay, P. \& De Oliveira, M., 2017. Species groups of Traumatomutilla André (Hymenoptera: Mutillidae), Insecta Mundi, 533: 1-38. 
Yu, D. S. K., van Achterberg. C. \& Horstmann, K., 2016. Taxapad 2016, Ichneumonoidea 2015. Database on flash-drive.
Whu, M. \& Valdivieso, I., 1999. Distribución y comportamiento de ocho especies de Trichogramma y Trichogrammatoidea(Hymenoptera:Trichogrammatidae) en el Perú. Revista Peruana de Entomología, 41: 61-68.

Apéndice 1.- Localidades de muestreo de colectas de himenópteros en la región Piura, Perú.

Appendix 1.- Sampling localities of himenopterans in the Piura region, Peru.

\begin{tabular}{|c|c|c|c|c|}
\hline Provincia & Localidad de muestreo & Coordenadas & Paisaje ecológico & Altura (msnm) \\
\hline \multirow[t]{8}{*}{ Piura } & Distrito Castilla & $05^{\circ} 11^{\prime} 05^{\prime \prime} \mathrm{S}-80^{\circ} 57^{\prime} 27^{\prime \prime} \mathrm{O}$ & BESII & 29 \\
\hline & Distrito Catacaos & $05^{\circ} 15^{\prime} 55^{\prime \prime} \mathrm{S}-80^{\circ} 40^{\prime} 30^{\prime \prime} \mathrm{O}$ & BESII & 29 \\
\hline & Universidad Nacional de Piura & $05^{\circ} 10^{\prime} 51^{\prime \prime} \mathrm{S}-80^{\circ} 37^{\prime} 31^{\prime \prime} \mathrm{O}$ & BESII & 29 \\
\hline & Universidad de Piura & $05^{\circ} 10^{\prime} 11^{\prime \prime} \mathrm{S}-80^{\circ} 36^{\prime} 51^{\prime \prime} \mathrm{O}$ & BESII & 29 \\
\hline & Distrito La Arena & $05^{\circ} 20^{\prime} 34^{\prime \prime} \mathrm{S}-80^{\circ} 42^{\prime} 30^{\prime \prime} \mathrm{O}$ & BESII & 29 \\
\hline & Distrito La Unión & $05^{\circ} 23^{\prime} 29^{\prime \prime} \mathrm{S}-80^{\circ} 44^{\prime} 58^{\prime \prime} \mathrm{O}$ & BESII & 29 \\
\hline & Distrito Miraflores & $05^{\circ} 16^{\prime} 66^{\prime \prime} \mathrm{S}-80^{\circ} 61^{\prime} 67^{\prime \prime} \mathrm{O}$ & BESII & 29 \\
\hline & Distrito Tambogrande & $04^{\circ} 55^{\prime} 05^{\prime \prime} \mathrm{S}-80^{\circ} 19^{\prime} 59^{\prime \prime} \mathrm{O}$ & BESII & 29 \\
\hline \multirow[t]{6}{*}{ Sullana } & Distrito Salitral & $04^{\circ} 51^{\prime} 27^{\prime \prime} \mathrm{S}-80^{\circ} 40^{\prime} 52^{\prime \prime} \mathrm{O}$ & BESII & 23 \\
\hline & Distrito Lancones & $04^{\circ} 38^{\prime} 27^{\prime \prime S}-80^{\circ} 32^{\prime} 55^{\prime \prime O}$ & BESc-BESm & $400-1500$ \\
\hline & Distrito Miguel Checa & $04^{\circ} 54^{\prime} \mathrm{S}-80^{\circ} 48^{\prime} \mathrm{O}$ & BESII & 23 \\
\hline & Distrito Querecotillo & $04^{\circ} 50^{\prime} 24^{\prime \prime S}-80^{\circ} 38^{\prime} 57^{\prime \prime O}$ & BESII & 23 \\
\hline & Distrito Ignacio Escudero & $04^{\circ} 50^{\prime} 35^{\prime \prime} \mathrm{S}-80^{\circ} 52^{\prime} 12^{\prime \prime} \mathrm{O}$ & BESII & 23 \\
\hline & Caserío Cabo Verde & $04^{\circ} 51^{\prime} 27^{\prime \prime} \mathrm{S}-80^{\circ} 40^{\prime} 52^{\prime \prime} \mathrm{O}$ & BESII & 23 \\
\hline \multirow[t]{2}{*}{ Paita } & Distrito de Paita & $04^{\circ} 04^{\prime} \mathrm{S}-81^{\circ} 06^{\prime} \mathrm{O}$ & BESII & $0-20$ \\
\hline & Distrito Vichayal & $04^{\circ} 51^{\prime} 55^{\prime \prime} \mathrm{S}-81^{\circ} 04^{\prime} 19^{\prime \prime} \mathrm{O}$ & BESII & $0-20$ \\
\hline \multirow[t]{5}{*}{ Talara } & Distrito Lobitos & $04^{\circ} 27^{\prime} 20^{\prime \prime} \mathrm{S}-81^{\circ} 16^{\prime} 57^{\prime \prime} \mathrm{O}$ & DESc-BESII & $0-100$ \\
\hline & Distrito de Los Órganos & $04^{\circ} 10^{\prime} 38^{\prime \prime} \mathrm{S}-81^{\circ} 07^{\prime} 29^{\prime \prime} \mathrm{O}$ & DESc & $0-10$ \\
\hline & Distrito de El Alto & $04^{\circ} 16^{\prime} 04^{\prime \prime S}-81^{\circ} 13^{\prime} 09^{\prime \prime O}$ & DESc & $0-10$ \\
\hline & Distrito de Pariñas & $04^{\circ} 34^{\prime} 52^{\prime \prime S}-81^{\circ} 16^{\prime} 29^{\prime \prime} \mathrm{O}$ & DESc-BESII & $0-10$ \\
\hline & Distrito de Máncora & $04^{\circ} 06^{\prime} 26^{\prime \prime} \mathrm{S}-81^{\circ} 02^{\prime} 50^{\prime \prime} \mathrm{O}$ & BESc-BESm & $750-1500$ \\
\hline \multirow[t]{5}{*}{ Sechura } & Distrito de Sechura & $05^{\circ} 29^{\prime} 37^{\prime \prime} \mathrm{S}-80^{\circ} 44^{\prime} 28^{\prime \prime} \mathrm{O}$ & DESc-BESII & $0-20$ \\
\hline & Distrito de Vice & $05^{\circ} 25^{\prime} \mathrm{S}-81^{\circ} 46^{\prime} \mathrm{O}$ & DESc-BESII & $0-20$ \\
\hline & Desierto de Sechura & $05^{\circ} 58^{\prime} \mathrm{S}-81^{\circ} 05^{\prime} \mathrm{O}$ & DESc & $0-5$ \\
\hline & Manglar de Vice & $05^{\circ} 30^{\prime} \mathrm{S}-80^{\circ} 54^{\prime} \mathrm{O}$ & DESc & $0-20$ \\
\hline & Distrito Cristo nos Valga & $05^{\circ} 29^{\prime} 37^{\prime \prime} \mathrm{S}-80^{\circ} 44^{\prime} 28^{\prime \prime} \mathrm{O}$ & BESII & 25 \\
\hline \multirow[t]{4}{*}{ Morropón } & Distrito de Chulucanas & $05^{\circ} 05^{\prime} \mathrm{S}-80^{\circ} 99^{\prime} \mathrm{O}$ & BESc-BESm & $300-1000$ \\
\hline & Distrito de Buenos Aires & $05^{\circ} 05^{\prime} 51^{\prime \prime} \mathrm{S}-80^{\circ} 09^{\prime} 44^{\prime \prime} \mathrm{O}$ & BESc-BESm & $300-1000$ \\
\hline & Caserío Piedra del Toro & $05^{\circ} 11^{\prime} \mathrm{S}-79^{\circ} 55^{\prime} \mathrm{O}$ & BESc-BESm & $300-1000$ \\
\hline & Distrito Santa Catalina de Mossa & $05^{\circ} 06^{\prime} 10^{\prime \prime} \mathrm{S} 79^{\circ} 53^{\prime} 06^{\prime \prime} \mathrm{O}$ & $\mathrm{BSI}$ & 1350 \\
\hline \multirow[t]{6}{*}{ Huancabamba } & Distrito de Huancabamba & $05^{\circ} 34^{\prime} 04^{\prime \prime} \mathrm{S}-79^{\circ} 31^{\prime} 26^{\prime \prime} \mathrm{O}$ & BESc-BESm & $300-1000$ \\
\hline & Distrito de Carmen de la Frontera & $05^{\circ} 08^{\prime} 54^{\prime \prime} \mathrm{S}-79^{\circ} 25^{\prime} 41^{\prime \prime} \mathrm{O}$ & $\mathrm{BHM}$ & $2450-3000$ \\
\hline & Distrito de Sondorillo & $05^{\circ} 24^{\prime} 07^{\prime \prime} \mathrm{S}-79^{\circ} 36^{\prime} 22^{\prime \prime} \mathrm{O}$ & $\mathrm{BSI}$ & $1650-2000$ \\
\hline & Distrito de Canchaque & $05^{\circ} 22^{\prime} 35^{\prime \prime} \mathrm{S}-79^{\circ} 36^{\prime} 23^{\prime \prime} \mathrm{O}$ & BSI-BHM & $1700-2200$ \\
\hline & Caserío de Pampaminas & $05^{\circ} 26^{\prime} 37^{\prime \prime} \mathrm{S}-79^{\circ} 45^{\prime} 26^{\prime \prime} \mathrm{O}$ & $\mathrm{BSI}$ & 1655 \\
\hline & Distrito de Sondor & $05^{\circ} 18^{\prime} 56^{\prime \prime} \mathrm{S}-79^{\circ} 24^{\prime} 36^{\prime \prime} \mathrm{O}$ & BSI & $1700-2000$ \\
\hline \multirow[t]{5}{*}{ Ayabaca } & Distrito Suyo & $04^{\circ} 30^{\prime} 45^{\prime \prime} \mathrm{S}-80^{\circ} 02^{\prime} 10^{\prime \prime} \mathrm{O}$ & BESc-BESm & $300-1000$ \\
\hline & Distrito de Jililí & $04^{\circ} 37^{\prime} 37^{\prime \prime} \mathrm{S}-79^{\circ} 56^{\prime} 43^{\prime \prime} \mathrm{O}$ & BESII & $300-1000$ \\
\hline & Caserío Yacupampa & $04^{\circ} 39^{\prime} \mathrm{S}-79^{\circ} 43^{\prime} \mathrm{O}$ & $\mathrm{BHM}$ & $2600-2950$ \\
\hline & Caserío Lagunas de Tunal & $04^{\circ} 47^{\prime} 23^{\prime \prime} \mathrm{S}-79^{\circ} 50^{\prime} 42^{\prime \prime} \mathrm{O}$ & $\mathrm{BHM}$ & 2800 \\
\hline & Caserio de Socchabamba & $04^{\circ} 36^{\prime} 35^{\prime \prime S}-79^{\circ} 42^{\prime} 34^{\prime \prime O}$ & $\mathrm{BHM}$ & $2300-3000$ \\
\hline
\end{tabular}

